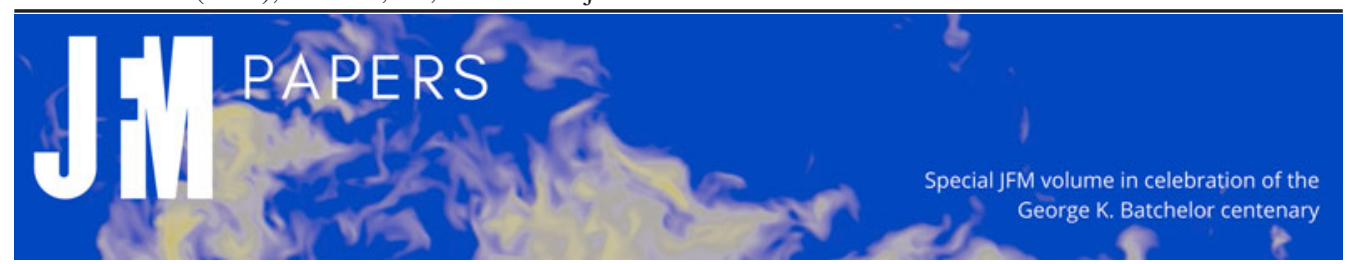

\title{
Interactions between scales in wall turbulence: phase relationships, amplitude modulation and the importance of critical layers
}

\author{
Ian Jacobi ${ }^{1}$, Daniel Chung ${ }^{2}$, Subrahmanyam Duvvuri ${ }^{3}$ and \\ Beverley J. McKeon ${ }^{4} \dagger$ \\ ${ }^{1}$ Faculty of Aerospace Engineering, Technion Israel Institute of Technology, Haifa 32000, Israel \\ ${ }^{2}$ Department of Mechanical Engineering, University of Melbourne, Victoria 3010, Australia \\ ${ }^{3}$ Department of Aerospace Engineering, Indian Institute of Science, Bengaluru 560 012, India \\ ${ }^{4}$ Graduate Aerospace Laboratories, California Institute of Technology, Pasadena, CA 91125, USA
}

(Received 20 March 2020; revised 23 July 2020; accepted 7 September 2020)

We present a framework for predicting the interactions between motion at a single scale and the underlying stress fluctuations in wall turbulence, derived from approximations to the Navier-Stokes equations. The dynamical equations for an isolated scale and stress fluctuations at the same scale are obtained from a decomposition of the governing equations and formulated in terms of a transfer function between them. This transfer function is closely related to the direct correlation coefficient of Duvvuri \& McKeon (J. Fluid Mech., vol. 767, 2015, R4), and approximately to the amplitude modulation coefficient described in Mathis et al. (J. Fluid Mech., vol. 628, 2009, pp. 311-337), by consideration of interactions between triadically consistent scales. In light of the agreement between analysis and observations, the modelling approach is extended to make predictions concerning the relationship between very-large motions and small-scale stress in the logarithmic region of the mean velocity. Consistent with experiments, the model predicts that the zero-crossing height of the amplitude modulation statistic coincides with the wall-normal location of the very large-scale peak in the one-dimensional premultiplied spectrum of streamwise velocity fluctuations, the critical layer location for the very large-scale motion. Implications of fixed phase relationships between small-scale stresses and larger isolated scales for closure schemes are briefly discussed.

Key words: turbulent boundary layers

$†$ Email address for correspondence: mckeon@caltech.edu 


\section{Jacobi, D. Chung, S. Duvvuri and B.J. McKeon}

\section{Large-scale motion in wall turbulence}

It is now clear that large-scale motion, that is, motion comprising scales larger than $\delta$, where $\delta$ is the boundary-layer height, channel half-height or pipe radius, plays an increasingly important role in wall turbulence as the Reynolds number increases (Kim \& Adrian 1999; Morrison et al. 2004; Hutchins \& Marusic 2007). In addition to contributing to the total energy, evidenced by the increasingly dominant very-large-scale peak at wavelengths of order $10 \delta$ in the premultiplied velocity spectrum, and the Reynolds shear stress, the very-large-scale motion also appears to modulate and organize the underlying small-scale motion. An aspect of this phenomenon is measured by the amplitude modulation statistic $\mathcal{R}$ (Mathis, Hutchins \& Marusic 2009a), a one-point statistic that measures the relative placement of small-scale activity to the large-scale motion. Near the wall, intense small-scale stresses accompany a large-scale higher momentum region and vice versa, corresponding to $\mathcal{R}>0$, but above a certain zero-crossing height, this relationship is reversed, $\mathcal{R}<0$. Interestingly, this height also tracks the wall-normal location of the very-large-scale spectral energy peak (Mathis et al. 2009a). The literature concerning amplitude modulation is extensive, thus we review here only the work directly relevant to the present contribution.

Bandyopadhyay \& Hussain (1984) and Jacobi \& McKeon (2013) used cross-correlation techniques to determine the temporal and spatial lead/lag information, respectively, related to the large and small scales in the amplitude modulation coefficient. Chung \& McKeon (2010) found similar information from the conditionally averaged large and small scales from LES, and Hutchins et al. (2011) from experiments.

More recently, Talluru et al. (2014) extended the conditional averaging studies of Chung \& McKeon (2010) and Hutchins et al. (2011) and earlier qualitative observations in Hutchins \& Marusic (2007) to characterize amplitude modulation effects in all three components of velocity with reference to the streamwise large-scale velocity. Ganapathisubramani et al. (2012) and Baars et al. (2015) inferred a coincident frequency modulation effect.

Schlatter \& Örlü (2010) and Mathis et al. (2011) observed similarity between the amplitude modulation statistic and the skewness of the streamwise velocity fluctuations, at least outside of the near-wall region; Duvvuri \& McKeon (2015) derived an exact analytical relationship between these two quantities and described it in terms of phase relationships and interactions between triadically consistent scales. The amplitude modulation coefficient can be interpreted as a dot product between the large-scale signal and the component of the envelope of small scales with the same frequency content, as noted by Chung \& McKeon (2010), such that as its varies from 1 to -1 the magnitude of the relative phase between large and small signals changes from 0 to $\pi$, with a zero amplitude modulation coefficient corresponding to signals that are $\pi / 2$ out of phase. This interpretation will prove central to the work that follows herein. Jacobi \& McKeon (2013) demonstrated that the amplitude modulation coefficient is dominated by the influence of the very-large-scale motions (VLSMs) by using cross-spectral analysis in a canonical zero pressure gradient turbulent boundary layer.

In terms of modelling the amplitude modulation effect, Marusic, Mathis \& Hutchins (2010) extended physical observations to provide predictions of near-wall activity based on measurements further from the wall, while Inoue et al. (2012) have utilized this model in combination with a large eddy simulation to extrapolate the behaviour of the streamwise velocity fluctuations to very high Reynolds numbers. Chernyshenko, Marusic \& Mathis (2012) have given a theoretical description of the effect of the large scales on the small scales in terms of a quasi-steady modulation of skin friction and small-scale activity, and 


\section{Interactions between scales in wall turbulence}

Mathis et al. (2013) extended these analyses to predict the time-varying skin friction from off-wall measurements.

McKeon \& Sharma (2010) and subsequent works by those authors and others presented a resolvent analysis in wall turbulence (in the former work in turbulent pipe flow) that predicts, amongst other things, important features of large-scale motion including scaling behaviour and physical structure. The energetically dominant structure is proposed to be controlled by the location of the critical layer, $y_{c}$, identified as the location where the local mean velocity is equal to the disturbance convection velocity. The predicted physical structure of the very-large-scale motion in the log region - weak upright regions of wall-normal momentum and inclined regions of approximately uniform streamwise momentum which have the potential to organize vortical structure (Sharma \& McKeon 2013 ) - are also in agreement with particle image velocimetry visualizations (e.g. Adrian, Meinhart \& Tomkins 2000) and conditional averaging, (e.g. Hutchins \& Marusic 2007; Chung \& McKeon 2010; Hutchins et al. 2011).

Recently, Dawson \& McKeon (2019) developed a semi-analytical procedure for approximating the shapes of the leading (most amplified) resolvent modes in quasi-parallel shear flows by consideration of wavepacket pseudoeigenmodes. Truncated asymptotic expansions of Airy functions were shown to provide an accurate representation of mode shapes associated with an approximation to the resolvent operator. Thus a template function for the wall-normal shape variation, a Gaussian amplitude profile with associated phase shift, with constants that can be determined by optimization can be used to give an excellent approximation to resolvent modes without the need for the computational cost associated with the discretization, matrix inversion and singular value decomposition steps in the traditional resolvent analysis. While this approach reduces computational cost, in this work the semi-analytical form for resolvent modes is of importance.

Extending the transfer function concepts underlying the analysis of McKeon \& Sharma (2010), and the treatment of the nonlinear forcing summarized in McKeon, Sharma \& Jacobi (2013), we present an analytical framework to predict the interactions between scales in wall turbulence that is capable of linking all three components of large- and small-scale velocity signals. To our knowledge, this is the first such approach derived directly from the full Navier-Stokes equations. Our focus in this paper is on streamwise velocity interactions and the modulating influence of large-scale motion in the log region on the underlying small-scale motion; however, the results have a broader reach in terms of a fundamental restriction on triadic interactions at all scales. The work supports the interpretation of the amplitude modulation coefficient as a reflection of the relative spatial organization of turbulent scales.

\section{Framework for scale interactions}

\subsection{Approach}

The incompressible Navier-Stokes equations are non-dimensionalized with friction velocity $u_{\tau}$ and outer length scale, $h$, corresponding to a half-channel height. The friction velocity is the relevant near-wall velocity scale in the region of the logarithmic layer which will be the focus of subsequent analysis. Following Reynolds \& Hussain (1972), we then decompose the flow field, described by the velocity $u_{i}$ and kinematic pressure $p$, into the mean $\overline{()}$, isolated single scale $\widetilde{()}$ and remaining turbulent ( )' components

$$
u_{i}=\bar{u}_{i}+\tilde{u}_{i}+u_{i}^{\prime}, \quad p=\bar{p}+\tilde{p}+p^{\prime} .
$$

Here, the isolated scale consists of a single turbulent scale and all other remaining turbulent activity is lumped into $u_{i}^{\prime}$. Such a decomposition is most easily conceptualized 


\section{Jacobi, D. Chung, S. Duvvuri and B.J. McKeon}

by invoking a narrow bandpass spectral filter around the wavenumber $\boldsymbol{k}_{\boldsymbol{f}}$; in this case the scale is cleanly defined in a spectral sense, but requires careful connection to observed very-large-scale motion. Here, we will adopt the simple narrow bandpass Fourier mode representation, but we argue later in $\S 3.1$ that this is sufficient for explaining the observed amplitude modulation coefficient and the underlying phase relationships. Thus, $\tilde{u}_{i}$ comprises modes with wavenumber $\boldsymbol{k}_{\boldsymbol{f}}$ while $u_{i}^{\prime}$ comprises modes with wavenumbers other than $\boldsymbol{k}_{\boldsymbol{f}}$. In what follows, we will define $\boldsymbol{k}_{\boldsymbol{f}}=\left(k_{x}, k_{z}, \omega\right)$, where $k_{x}$ and $k_{z}$ are real wavenumbers in the two spatial (wall-parallel) directions and $\omega$ is the real angular frequency, effectively a single scale in a triple Fourier decomposition. Substituting $(2.1 a, b)$ into the Navier-Stokes equations, that is,

$$
\frac{\partial u_{i}}{\partial t}+u_{j} \frac{\partial u_{i}}{\partial x_{j}}=-\frac{\partial p}{\partial x_{i}}+\frac{1}{R e} \frac{\partial^{2} u_{i}}{\partial x_{j}^{2}}, \quad \frac{\partial u_{i}}{\partial x_{i}}=0,
$$

where $R e \equiv u_{\tau} h / v$ and $v$ is the kinematic viscosity, and then applying the narrow bandpass filter at $\boldsymbol{k}_{\boldsymbol{f}}$, we obtain the dynamical equation for the isolated-scale motion at $\boldsymbol{k}_{\boldsymbol{f}}$

$$
\frac{\partial \tilde{u}_{i}}{\partial t}+\bar{u}_{j} \frac{\partial \tilde{u}_{i}}{\partial x_{j}}+\tilde{u}_{j} \frac{\partial \bar{u}_{i}}{\partial x_{j}}+\frac{\partial \tilde{p}}{\partial x_{i}}-\frac{1}{R e} \frac{\partial^{2} \tilde{u}_{i}}{\partial x_{j}^{2}}=-\frac{\partial \tilde{r}_{i j}}{\partial x_{j}} \equiv \tilde{f}_{i}, \quad \frac{\partial \tilde{u}_{i}}{\partial x_{i}}=0,
$$

where $\tilde{r}_{i j}=\widetilde{u_{i}^{\prime} u_{j}^{\prime}}$, the filtered fluctuation of the background mean stress, $\bar{r}_{i j}=\overline{u_{i}^{\prime} u_{j}^{\prime}}$, at the isolated scale (i.e. with wavenumber $\boldsymbol{k}_{f}$ ), with contributions from fluctuations with $\boldsymbol{k} \neq \boldsymbol{k}_{\boldsymbol{f}}$. The dynamical equation for $\tilde{r}_{i j}$ (see Reynolds \& Hussain 1972) is

$$
\frac{\partial \tilde{r}_{i j}}{\partial t}+\bar{u}_{k} \frac{\partial \tilde{r}_{i j}}{\partial x_{k}}+\tilde{u}_{k} \frac{\partial \bar{r}_{i j}}{\partial x_{k}}+\tilde{r}_{j k} \frac{\partial \bar{u}_{i}}{\partial x_{k}}+\tilde{r}_{i k} \frac{\partial \bar{u}_{j}}{\partial x_{k}}+\bar{r}_{j k} \frac{\partial \tilde{u}_{i}}{\partial x_{k}}+\bar{r}_{i k} \frac{\partial \tilde{u}_{j}}{\partial x_{k}}-\frac{1}{R e} \frac{\partial^{2} \tilde{r}_{i j}}{\partial x_{k}^{2}}=\tilde{g}_{i j},
$$

where

$$
\tilde{g}_{i j}=-\frac{\partial}{\partial x_{k}} \widetilde{u_{i}^{\prime} u_{j}^{\prime} u_{k}^{\prime}}-\widetilde{u_{j}^{\prime} \frac{\partial p^{\prime}}{\partial x_{i}}}-\widetilde{u_{i}^{\prime} \frac{\partial p^{\prime}}{\partial x_{j}}}-\frac{2}{\operatorname{Re}} \widehat{\frac{\partial u_{i}^{\prime}}{\partial x_{k}} \frac{\partial u_{j}^{\prime}}{\partial x_{k}}} .
$$

Note the slight difference between the equivalent phase-averaged results of Reynolds \& Hussain (1972) and the effective Fourier decomposition of (2.3) and (2.4), namely, that the difference and product terms, i.e. the mean and $2 \boldsymbol{k}_{\boldsymbol{f}}$ contributions, arising from phase averaging do not appear here as only the fluctuations at a single scale are being considered. The left-hand side of (2.4a) contains only linear terms, but the unclosed terms on the right renders it intractable for analysing the interaction between the isolated-scale motion $\tilde{u}_{i}$ and stress fluctuation $\tilde{r}_{i j}$ at first glance.

The linear operator containing the mean turbulent velocity profile in (2.3), the resolvent, describes many essential features of wall turbulence, including very-large-scale motion. In so-called resolvent analysis, the right-hand side, $\tilde{f}_{i}$, is modelled as an external forcing to the linear operator at each $\boldsymbol{k}_{f}=\left(k_{x}, k_{z}, \omega\right)$ combination. The linear operator then responds to the forcing by preferentially amplifying certain modes above others. The most amplified (first) of these 'velocity response modes', or the mode most receptive to disturbances, as identified using the gain-based singular value decomposition, are then interpreted to be the likely candidates for turbulent motion observed in nature. For the sake of brevity, the reader is referred to McKeon (2017) for details of the full formulation of the resolvent analysis. Channel flow is selected because the formulation for a fully developed flow is simpler than for a spatially growing (or locally parallel) turbulent boundary layer, although 


\section{Interactions between scales in wall turbulence}

we also make a comparison with turbulent boundary-layer results, internal versus external flow boundary condition considerations notwithstanding.

Extending the original resolvent analysis, we wish to consider the interaction between a given energetic motion and stress fluctuation at the same scale by augmenting the linear operator for $\tilde{u}_{i},(2.3 a, b)$, with the linear operator for $\tilde{r}_{i j},(2.4 a)$. Relative to the approach of McKeon \& Sharma (2010), this constitutes adding an equation to supply the appropriate structure for $\tilde{f}_{i}$. In the same vein, we model the nonlinear and unclosed terms $\tilde{g}_{i j}$ as external forcing. Owing to the increased complexity of this proposed approach, it seems prudent to first consider a simpler version of the scale interaction equations, namely by setting $\tilde{g}_{i j}=0$. In effect, this can be considered as retaining only the part of $\tilde{r}_{i j}$ that is directly coupled to $\tilde{u}_{i}$; the part of $\tilde{r}_{i j}$ that responds to $\tilde{g}_{i j}$ is treated as uncorrelated to $\tilde{u}_{i}$ and therefore vanishes when joint statistics with $\tilde{u}_{i}$ are taken. Further manipulation of terms $2-4$ in $\tilde{g}_{i j}$ is likely to result in their expression in terms of $\tilde{r}_{i j}$, particularly in light of the Biot-Savart relationship and the characterization of the pressure associated with each response mode by Luhar, Sharma \& McKeon (2014), which was shown to constitute the fast pressure, or that part of the pressure that is directly correlated with the mode velocity field, and the observations of amplitude modulation of the dissipation, the last term in $\tilde{g}_{i j}$, by Guala, Metzger \& McKeon (2011). However, this is beyond the scope of the current problem. We focus on this simplified problem with a view to demonstrating the origin of the amplitude modulation of the small scales by the large scales described in $\S 1$.

Our approach is to formulate and analyse the transfer function between the isolated scale and small-scale stress implied by $(2.4 a)$. In $\S 3$, we formulate the transfer function between the analytically inspired modal formulations of small-scale stresses and isolated scales in the vicinity of a critical layer, and determine the phase difference between the two modes. In light of the agreement between our analysis and observations, we extend the modelling approach to make predictions concerning the relationship between very-large-scale motions and small-scale stress in the logarithmic region of the mean velocity in $\S 4$.

\subsection{Set-up for wall turbulence}

Although formally applicable only for channel flows, the following analysis can also be extended to pipe and boundary-layer flows under the parallel flow assumption. A comparison between flows is likely to be most valid in the near-wall log and viscous regions, that is, where differences in flow geometry are negligible. We specialize the linear system, (2.4a) with $\tilde{g}_{i j}=0$, to wall turbulence

$$
\bar{u}=\bar{u}(y), \quad\{\bar{v}, \bar{w}\}=0,
$$

where $x, y$ and $z$ or $u, v$ and $w$ are the streamwise, wall-normal and spanwise coordinates or velocities. The mean stresses generated by the fluctuating scales are given by

$$
\left\{\bar{r}_{x x}, \bar{r}_{y y}, \bar{r}_{z z}, \bar{r}_{x y}\right\}=\left\{\bar{r}_{x x}, \bar{r}_{y y}, \bar{r}_{z z}, \bar{r}_{x y}\right\}(y), \quad\left\{\bar{r}_{x z}, \bar{r}_{y z}\right\}=0 ;
$$

the zeroes are from statistical symmetry. Velocity and stress fluctuations at the scale of interest are written as

$$
\left\{\tilde{u}_{i}, \tilde{r}_{i j}\right\}(x, y, z, t)=\left\{\tilde{U}_{i}, \tilde{R}_{i j}\right\}\left(y ; k_{x}, k_{z}, \omega\right) \exp \left(\mathrm{i}\left(k_{x} x+k_{z} z-\omega t\right)\right)+\text { c.c. },
$$

where the complex $\tilde{U}_{i}$ and $\tilde{R}_{i j}$ can be written in terms of a magnitude and phase as $\tilde{U}_{i}=$ $\left|\tilde{U}_{i}\right| \mathrm{e}^{\mathrm{i} \phi_{U_{i}}}$ and $\tilde{R}_{i j}=\left|\tilde{R}_{i j}\right| \mathrm{e}^{\mathrm{i} \phi_{R_{i j}}}$, and c.c. represents the complex conjugate of the preceding terms. 
In this work, we focus our attention on $(2.4 a)$. Substituting $(2.5 a, b),(2.6 a, b)$ and $(2.7)$ in $(2.4 a)$, we obtain for each $\left(k_{x}, k_{z}, \omega\right)$,

$$
\boldsymbol{A R}+\boldsymbol{B U}=0
$$

where

$$
\begin{aligned}
& \boldsymbol{A}=\mathrm{i}\left(-\omega+k_{x} \bar{u}\right)\left[\begin{array}{cccccc}
1 & 0 & 0 & 2 \gamma & 0 & 0 \\
0 & 1 & 0 & 0 & 0 & 0 \\
0 & 0 & 1 & 0 & 0 & 0 \\
0 & \gamma & 0 & 1 & 0 & 0 \\
0 & 0 & 0 & 0 & 1 & \gamma \\
0 & 0 & 0 & 0 & 0 & 1
\end{array}\right]-\operatorname{Re}^{-1}\left(\mathrm{~d}^{2}-k^{2}\right) \boldsymbol{I} ; \quad \boldsymbol{R}=\left[\begin{array}{c}
\tilde{R}_{x x} \\
\tilde{R}_{y y} \\
\tilde{R}_{z z} \\
\tilde{R}_{x y} \\
\tilde{R}_{x z} \\
\tilde{R}_{y z}
\end{array}\right] \\
& \boldsymbol{B}=\left[\begin{array}{ccc}
2 \bar{r}_{x x} \mathrm{i} k_{x}+2 \bar{r}_{x y} \mathrm{~d} & \bar{r}_{x x, y} & 0 \\
0 & \bar{r}_{y y, y}+2 \bar{r}_{x y} \mathrm{i} k_{x}+2 \bar{r}_{y y} \mathrm{~d} & 0 \\
0 & \bar{r}_{z z, y} & 2 \bar{r}_{z z} \mathrm{i} k_{z} \\
\bar{r}_{x y} \mathrm{i} k_{x}+\bar{r}_{y y} \mathrm{~d} & \bar{r}_{x y, y}+\bar{r}_{x x} \mathrm{i} k_{x}+\bar{r}_{x y} \mathrm{~d} & 0 \\
\bar{r}_{z z} \mathrm{i} k_{z} & 0 & \bar{r}_{x x} \mathrm{i} k_{x}+\bar{r}_{x y} \mathrm{~d} \\
0 & \bar{r}_{z z} \mathrm{i} k_{z} & \bar{r}_{x y} \mathrm{i} k_{x}+\bar{r}_{y y} \mathrm{~d}
\end{array}\right] ; \quad \boldsymbol{U}=\left[\begin{array}{c}
\tilde{U} \\
\tilde{V} \\
\tilde{W}
\end{array}\right]
\end{aligned}
$$

$\gamma=(\mathrm{d} \bar{u} / \mathrm{d} y) /\left[\mathrm{i}\left(-\omega+k_{x} \bar{u}\right)\right] ; \overline{(~)}_{, y} \equiv \mathrm{d} \overline{()} / \mathrm{d} y ; \mathrm{d} \equiv \mathrm{d} / \mathrm{d} y ; k^{2}=k_{x}^{2}+k_{z}^{2} ;$ and $\boldsymbol{l}$ is the identity matrix. The denominator of $\gamma$ has special significance in the vicinity of a critical layer, i.e. the wall-normal location, $y_{c}$, where the streamwise propagation velocity of a motion of scale $\boldsymbol{k}_{f}$ is equal to the local mean velocity,

$$
-\omega+k_{x} \bar{u}\left(y_{c}\right) \equiv 0 \quad \text { such that } \begin{cases}\bar{u}<\omega / k_{x} & \text { if } y<y_{c} \\ \bar{u}=\omega / k_{x} & \text { if } y=y_{c} \\ \bar{u}>\omega / k_{x} & \text { if } y>y_{c}\end{cases}
$$

Given $k_{x}$ and $k_{z}$, one could, in principle, discretize $\mathrm{d}$ and insert $\tilde{U}_{i}$ and $\omega$ from an earlier analysis (e.g. McKeon \& Sharma 2010) to obtain the response $\tilde{R}_{i j}$. However, unlike the earlier analysis of the velocities, the discretization of $\boldsymbol{B}$ involves measuring or approximating all of the average Reynolds stresses, $\bar{r}_{i j}$, also. Because it is not clear how sensitive the relationship between $\boldsymbol{U}$ and $\boldsymbol{R}$ is to the details of the Reynolds stresses, we approach the problem analytically, in order to draw conclusions based on a minimum of empirical measurements while also establishing the extent to which detailed measurements may be important for any future, discretized analysis. Here, we examine the implications of the analytical transfer function between $\boldsymbol{R}$ and $\boldsymbol{U}$ of (2.8) by employing a semi-analytical form for the first resolvent modes. In particular, we focus our effort on the relationship between the streamwise velocity components, which have been the topic of extended study through experimental observations in canonical wall turbulence, and seek to identify the transfer function between the isolated scale $\tilde{U}$ and corresponding fluctuating streamwise 
stress, $\tilde{R}_{x x}$. Introducing a relative phase between $\tilde{R}_{x x} \equiv \tilde{R}_{11}$ and $\tilde{U} \equiv \tilde{U}_{1}$,

$$
\frac{\tilde{R}_{x x}(y)}{\tilde{U}(y)}=\frac{\left|\tilde{R}_{x x}(y)\right|}{|\tilde{U}(y)|} \mathrm{e}^{\mathrm{i} \varphi(y)},
$$

where $\varphi \equiv \arg \tilde{R}_{x x}-\arg \tilde{U}=\arg \left(\tilde{R}_{x x} \tilde{U}^{*}\right) \equiv \phi_{R}-\phi_{U}$, where $*$ denotes the complex conjugate. Note that the relative phase $\varphi$ is defined with respect to the spatial variables such that it has the opposite sense to the phase described relative to the temporal domain in e.g. Jacobi \& McKeon (2013).

\subsection{Very-large-scale motion as the isolated scale, $\tilde{U}$}

We consider a three-dimensional isolated scale that is representative of the structure of a VLSM and turn our attention to scale interactions occurring in the log region of wall turbulence. Observations of VLSMs in the literature (e.g. Hutchins \& Marusic 2007; Chung \& McKeon 2010; Hutchins et al. 2011) have identified the appropriate streamwise and spanwise wavenumbers to be $k_{x} \approx 1$ and $k_{z} \approx \pm 6$, corresponding to wavelengths of approximately six and one times the outer length scale, respectively, and a structure convecting in the $x$-direction. The appropriate frequency (or, equivalently, convection velocity) can be determined with reference to the channel flow results of Mathis et al. (2009b) at $R e_{\tau}=3000$. The locus of the outer (large scale) peak energy has been identified as corresponding to $y=3.9 R e_{\tau}^{-1 / 2}$, although there remains some uncertainty about this particular form (see Vallikivi, Ganapathisubramani \& Smits 2015). McKeon \& Sharma (2010) have discussed the effectiveness of a single resolvent mode as a proxy for the real VLSM; extending this approach, the location of the outer peak energy corresponds to the VLSM critical layer, such that the convection velocity is given by the local mean velocity at this wall-normal location.

\subsection{Phase relationship between the streamwise velocity of the isolated scale and corresponding fluctuating stress via correlation coefficients}

The phase relationship between the streamwise component of the isolated scale, $\tilde{u}$, and the stress fluctuation at the same isolated scale, $\tilde{r}_{x x}$, can also be characterized using experimental or numerical observations and a direct correlation coefficient (Duvvuri \& McKeon 2015; Jacobi \& McKeon 2017)

$$
\Phi(y)=\frac{\left\langle\tilde{u} \tilde{r}_{x x}\right\rangle}{\left\langle(\tilde{u})^{2}\right\rangle^{1 / 2}\left\langle\left(\tilde{r}_{x x}\right)^{2}\right\rangle^{1 / 2}},
$$

where \langle\rangle is the inner product. It can then be shown that

$$
\Phi=\cos \varphi
$$

that is, $\Phi$ is directly related to the relative phase between $\tilde{R}_{x x}$ and $\tilde{U}(2.10)$. It does not contain explicit information about their relative magnitudes due to the normalization, nor the sense of the phase relationship due to the symmetry of the cosine function.

The more common amplitude modulation coefficient, $\mathcal{R}$, was derived (e.g. Bandyopadhyay \& Hussain 1984; Mathis et al. 2009a) in terms of the correlation between 


\section{Jacobi, D. Chung, S. Duvvuri and B.J. McKeon}

a large-scale signal, $u_{L}$, defined using a filter in wavenumber space at $k_{x}=k_{\zeta}$, where

$$
u(y, t)=u_{L}(y, t)+u_{S}(y, t),
$$

and the envelope, $\mathcal{E}_{L}$, of the small-scale turbulence signal, $u_{S}$, filtered at the same wavenumber, i.e.

$$
\mathcal{R}=\frac{\left\langle u_{L} \mathcal{E}_{L}\right\rangle}{\left\langle u_{L}^{2}\right\rangle^{1 / 2}\left\langle\mathcal{E}_{L}^{2}\right\rangle^{1 / 2}} .
$$

Section 4 describes the relationship between $\mathcal{R}$, the stress fluctuation $\tilde{r}_{x x}$ and the isolated scale $\tilde{u}$, and argues that the isolated-scale and the stress-fluctuation terms are representative of the envelopes of large- and small-scale fluctuations. Therefore, the analysis here in terms of $\tilde{U}$ and $\tilde{R}_{x x}$ is relevant also to the filtered, amplitude modulation analysis.

Qualitatively, correlation coefficients such as $\Phi$ and $\mathcal{R}$ can be interpreted in terms of triadically consistent scales: non-zero contributions arise only from stress fluctuations at the wavenumber of the isolated scale in $\Phi$, or from small-scale stress fluctuations associated with the entire spectral content of the large-scale velocity signal, $u_{L}$, in $\mathcal{R}$. To reiterate, then, the direct correlation coefficient, $\Phi$, describes the relationship between the isolated scale and that portion of the stress fluctuation $r_{x x}$ that is triadically consistent with $\tilde{U}$, namely $\tilde{R}_{x x}$. By contrast, the amplitude modulation coefficient considers only the small-scale stress component that is triadically consistent with the entire spectral content of the large-scale signal, $u_{L}$. A formal description of this interpretation is given in Duvvuri \& McKeon (2015).

\section{Scale interactions for three-dimensional isolated scales}

\subsection{Transfer function including viscous effects}

Our ultimate goal is the investigation of the amplitude modulation effect in canonical wall turbulence. This has been shown to be dominated by the VLSM (Jacobi \& McKeon 2013), i.e. we will later hypothesize that the VLSM can be modelled by an isolated scale in this analysis. However, we begin by considering the scale interactions corresponding to a single, truly isolated very large scale. We focus our effort on the relationship between streamwise velocity components, which has been the topic of extended study through experimental observations in canonical wall turbulence, and identify the transfer function between the isolated scale, $\tilde{U}$, and corresponding fluctuating streamwise stress, $\tilde{R}_{x x}$. The transfer function indicates the relative magnitudes and phases between the isolated scale and fluctuating stresses. In general, the phase difference between two scales can be used to reconstruct the relative spatial orientation between them. If the orientation of an isolated, large-scale motion, i.e. its downstream inclination angle, is known, then the phase difference between that large-scale and another scale indicates the relative inclination of the second scale with respect to the first. Here, the transfer function can provide a picture of the relative spatial orientation of these large-scale motions and fluctuating stress in wall-bounded flows.

Because the structure of the large-scale motions is hypothesized to be intrinsically connected to the location of the critical layer for VLSMs, where the outer energy peak is observed in premultiplied spectral maps, we focus the transfer function analysis on VLSMs in the spatial vicinity of their critical layer.

The critical layer is the viscous region of flow that resolves the singularity in the inviscid Rayleigh equation which occurs when the phase speed of a neutrally stable 


\section{Interactions between scales in wall turbulence}

velocity mode equals the local mean convection velocity of the flow in which it propagates. The viscous critical layer behaviour applies to small amplitude velocity modes; for large amplitude disturbances, nonlinear critical behaviour can resolve the singularity in the absence of viscosity, as detailed in Haberman (1976). Therefore, we must assume that the nonlinear forcing contained in the $\tilde{f}_{i}$ term in (2.3) is small. Lin (1955) showed through asymptotic analysis that the thickness of the linear critical layer, $\epsilon$, scales as $\left(k_{x} R e\right)^{-1 / 3}$, thus approaching zero as $\left(k_{x} R e\right) \rightarrow \infty$. For convenience, we include the local velocity gradient at the critical point, $\mathrm{d} \bar{u}_{c}$, in the definition of the critical layer thickness

$$
\epsilon=\left(k_{x} \mathrm{~d} \bar{u}_{c} R e\right)^{-1 / 3} .
$$

For large wavelength velocity modes, the critical layer forms a distinct region of the flow, separate from the viscous wall layer.

However, because viscosity is not negligible in the critical layer, the Reynolds number dependent terms in (2.8b) must be preserved, which means the $\boldsymbol{A}$ matrix operator acting on the $\tilde{R}_{i j}$ terms is invertible upon discretization of the d operator, but no simple transfer function between arbitrary $\tilde{U}$ and $\tilde{R}_{i j}$ can be determined analytically. In order to obtain a transfer function applicable in the vicinity of the viscous critical layer, we must therefore assume that the functional form of the stress fluctuations, $\tilde{R}_{i j}$, is modal.

In order to approximate a modal representation of the stress fluctuations, we begin with the representation of a single, isolated velocity scale. One such representation is given in the work of Dawson \& McKeon (2019), who showed that the leading streamwise modes associated with the incompressible Navier-Stokes resolvent operator can be approximated in analytical form, in the limit of long wavelength disturbances, $k \ll 1$ (using the outer non-dimensionalization), as

$$
\tilde{U}=M_{U} \exp \left[-\mathrm{i} \alpha_{U} \frac{\left(y-y_{m}\right)}{\epsilon}-\beta_{U}\left(\frac{y-y_{m}}{\epsilon}\right)^{2}\right],
$$

for positive, $O(1)$ coefficients $\alpha_{U}=\alpha_{U}\left(k_{z}, k_{z}\right)$ and $\beta_{U}=\beta_{U}\left(k_{z}, k_{z}\right)$ that depend on the streamwise wavenumber $k_{x}$ and the wall-parallel wavenumber magnitude $k$, with $M_{U}=M_{U}\left(k_{x}, k_{z}\right)$ the amplitude of the mode peak; $y_{m}$ denotes the location of the modal maximum, dictated by the wave speed of the mode, $\omega / k_{x}$. The asymptotic form of (3.2) consists of a Gaussian profile whose width scales with the size of the critical layer, $\epsilon$. This general model was shown to be quite robust, applying over an unexpectedly large range of streamwise wavenumbers, especially in the vicinity of the critical layer itself. However, the model was not formally developed to describe the wall-normal velocity component, which is needed here for formulating the fluctuating stresses, and thus further assumptions will be necessary for this component. Other models are also possible; the work of Dawson \& McKeon (2019) is used by way of example. The validity of the following analysis depends on this particular choice of model only insofar as the length scales associated with the mode shapes scale on the critical layer thickness, $\epsilon$.

The fluctuating stress, $\tilde{R}_{i j}$, associated with the specific triadic interaction that includes the isolated large scale, $\tilde{U}$, can be constructed by multiplication of the two remaining members of that triad, each obtained from a distinct, narrow, band-pass filter of the governing equations. For the streamwise, normal Reynolds stress, we simply multiply two instances of the Gaussian model in (3.2), which results in another Gaussian model for the 
stress with $i=j=x$

$$
\tilde{R}_{i j}=M_{i j} \exp \left[-\mathrm{i} \alpha_{i j} \frac{\left(y-y_{m, i j}\right)}{\epsilon}-\beta_{i j}\left(\frac{y-y_{m, i j}}{\epsilon}\right)^{2}\right],
$$

where the coefficients $\alpha_{i j}=\alpha_{i j}\left(k_{x}, k_{z}\right)$ and $\beta_{i j}=\beta_{i j}\left(k_{x}, k_{z}\right)$ reflect the width and phase variations of the resulting stress, which depend on the triadic composition of the two isolated mode wavenumbers through their respective values of $\alpha_{U}$ and $\beta_{U}$.

In order to construct the $\tilde{R}_{x y}$ and $\tilde{R}_{y y}$ fluctuating stresses, we need to first modify (3.2) to describe the wall-normal component, $\tilde{V}$. The primary distinguishing features of the wall-normal component are: (i) the lack of phase variation across the region of the critical layer, as discussed in more detail in $\$ 3.2$; and (ii) a taller Gaussian profile than the streamwise component. Therefore, we assume that $\alpha_{V} \approx 0$ and that the profile of $\tilde{V}$ can be approximated by a constant-phase Gaussian, at least in the vicinity of the critical layer, with $\beta_{V}<\beta_{U}$ to enforce a taller profile. Performing the same multiplication within the triad described above, $\tilde{R}_{x y}$ takes the same form as (3.3), since the phase variation represented by $\alpha_{x y}$ is preserved by the streamwise component, and $\tilde{R}_{y y}$ also takes that form but with $\alpha_{y y} \approx 0$, since neither wall-normal component includes phase variation, and also $\beta_{y y}<\beta_{x x}$.

We apply the formulation of $\tilde{R}_{i j}$ in (3.3) to show that when the viscous part of the $\boldsymbol{A}$ matrix operator acts on $\tilde{R}_{i j}$, the result is simply proportional to $\tilde{R}_{i j}$ itself, i.e.

$$
-\left(\mathrm{d}^{2}-k^{2}\right) \tilde{R}_{i j}=\underbrace{\left[k^{2}+2 \frac{\beta_{i j}}{\epsilon^{2}}+\left(\frac{\alpha_{i j}}{\epsilon}-\mathrm{i} \frac{2 \beta_{i j}}{\epsilon^{2}}\left(y-y_{m, i j}\right)\right)^{2}\right]}_{\ell_{i j}^{-2}(y)} \tilde{R}_{i j},
$$

where we define a wavenumber-dependent length scale $\ell_{i j}$ to represent the local radius of curvature of the stress profile. See figure 1 for a sketch of the physical representation of the isolated mode and stress, which identifies the relationship between, for example, $\phi_{U}$ and $\alpha_{U}$.

Substituting the modal form of the stress into the matrix operator $\boldsymbol{A}$ renders the system analytically invertible and thus the transfer function is obtained by solving

$$
\boldsymbol{R}=-\boldsymbol{A}^{-1} \boldsymbol{B} \boldsymbol{U}
$$

to arrive at the direct relation between the streamwise component of stress, $\tilde{R}_{x x}$, and the large scale $\tilde{U}$. The full transfer function, written in terms of a general modal stress, is presented in appendix A, specifically (A3).

\subsection{Simplified transfer function near the critical layer}

The analytical transfer function, including viscosity, is then simplified by expanding it about a point $y$ in the vicinity of the critical point, $y_{c}$ (where the local mean velocity matches the wave speed, according to (2.9)), by writing

$$
\left(-\omega+k_{x} \bar{u}\right) \approx k_{x} \mathrm{~d} \bar{u}_{c} \Delta y=\epsilon^{-3} \operatorname{Re}^{-1} \Delta y,
$$

where $y=y_{c}+\Delta y, \Delta y / \epsilon \ll 1$ and the definition of the critical layer thickness, $\epsilon$, is given by (3.1). Substituting into $\gamma$, we obtain

$$
\gamma=\frac{-\mathrm{i} \mathrm{d} \bar{u}}{\left(-\omega+k_{x} \bar{u}\right)} \approx \frac{-\mathrm{i} \mathrm{d} \bar{u}}{k_{x} \mathrm{~d} \bar{u}_{c} \Delta y} \approx \frac{-\mathrm{i}}{\left(k_{x} \Delta y\right)} \frac{y_{c}}{y},
$$



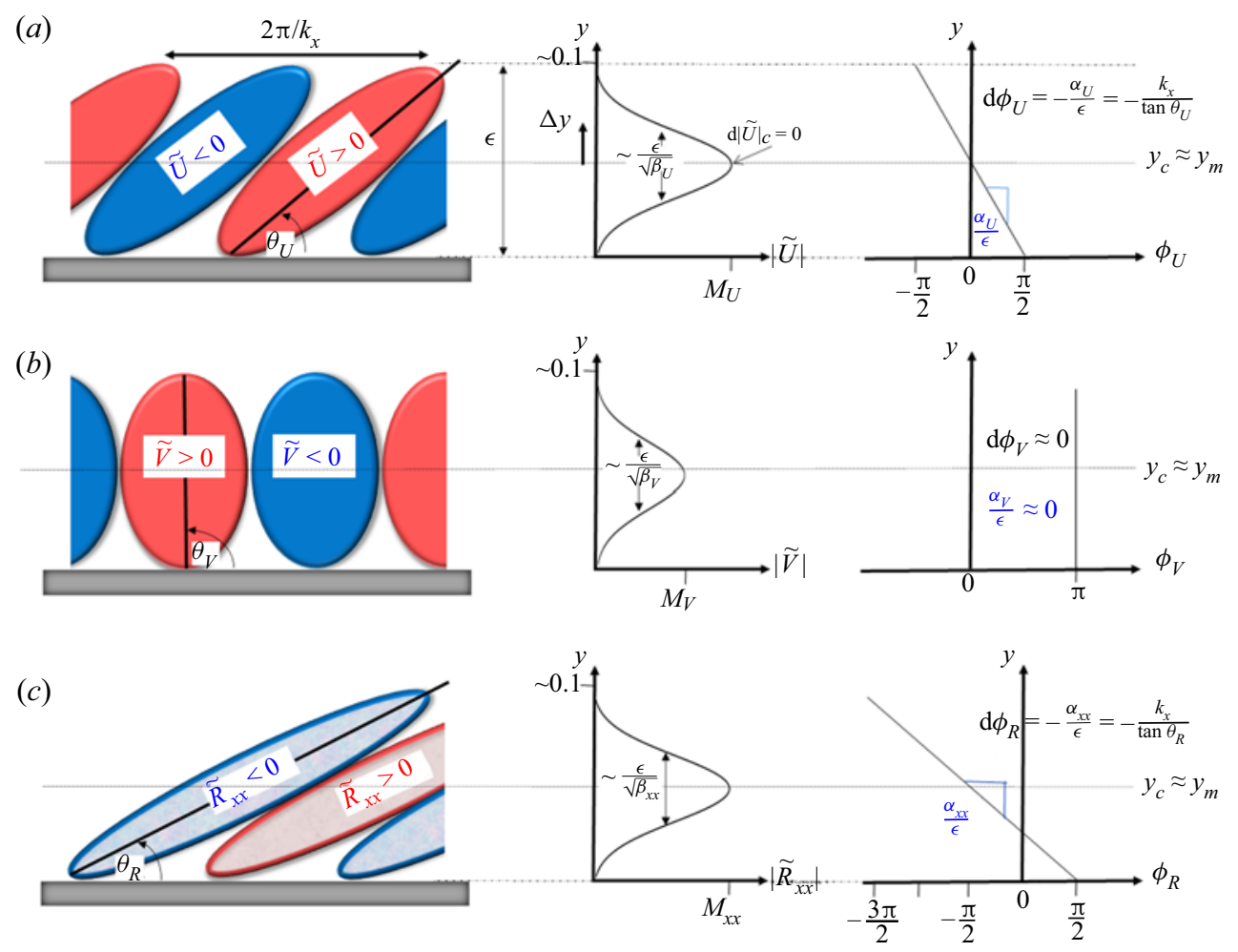

Figure 1. A schematic of the relevant length scales associated with the large- and small-scale motions in the vicinity of the critical layer. ( $a$ ) The streamwise component of the isolated large-scale mode can be represented by (3.2), with mode width $\beta_{U}$ and phase slope, near its centre $y_{m}$ of $-\alpha_{U} / \epsilon$, resulting in a downstream inclination $\theta_{U}$. (b) The wall-normal component of the isolated large-scale mode. Here, the key assumption is that the phase variation across the critical layer, $\mathrm{d} \phi_{V}$ is negligible and that $\mathrm{d}|\tilde{V}| /|\tilde{V}| \lesssim k_{x}$. (c) The streamwise stress component, which can also be represented by (3.3). At the wall, the streamwise stress shares the same phase as the isolated large scale. At the critical layer, the large-scale phase is 0 and the small-scale phase is $-\pi / 2$, such that $\varphi=\phi_{R}-\phi_{U}=-\pi / 2$, consistent with (3.14).

where we assume that the velocity profile is logarithmic and thus $\mathrm{d} \bar{u}=1 /(\kappa y)$ and $\mathrm{d} \bar{u}_{c}=$ $1 /\left(\kappa y_{c}\right)$, where $\kappa$ is the von Kármán constant. ( $\mathrm{d} \bar{u}$ can also be evaluated at the critical layer itself, but we retain the $y$-dependence for generality.)

Near the critical layer, we also neglect the $y$-dependence in the stress curvature length scale, $\ell_{i j}$, by assuming that the critical layer occurs near the peak of the fluctuating stress profile. For the streamwise, normal and shear stresses, this means $\mathrm{d}\left|\tilde{R}_{i j}\right| /\left|\tilde{R}_{i j}\right| \ll\left|\mathrm{d} \phi_{R_{i j}}\right|$. Using the analytical model in (3.3), this assumption is equivalent to the claim that the distance between the critical layer and the modal maximum is much smaller than the layer itself, i.e. $\left|y_{c}-y_{m, i j}\right| / \epsilon \ll \alpha_{i j} /\left(2 \beta_{i j}\right)$, where $\Delta y$ is assumed arbitrarily small, for simplicity. For $\tilde{R}_{y y}$, where $\alpha_{y y} \approx 0$, we assume instead that $\left|y_{c}-y_{m, y y}\right| / \epsilon \ll \sqrt{1 /\left(2 \beta_{y y}\right)}$. Because $\beta_{y y}<\beta_{x x}$, this assumption implies that the distance between the centre of $\tilde{R}_{y y}$ and the critical layer may be greater than in the case of $\tilde{R}_{x x}$. Applying these assumptions about $\ell_{i j}$, we eliminate the final term in the square brackets of (3.4) and the radius of curvature reduces to

$$
\ell_{i j}^{2}=\frac{\epsilon^{2}}{(k \epsilon)^{2}+2 \beta_{i j}+\alpha_{i j}^{2}},
$$




\section{Jacobi, D. Chung, S. Duvvuri and B.J. McKeon}

where $\ell_{i j}>0$ is now real. Assuming that $(k \epsilon)$ is small at high Reynolds number (i.e. $k_{z} \epsilon \ll 1$ for small $k_{x}$ ) and that the stress modes scale on the critical layer thickness, such that the $\alpha_{i j}$ and $\beta_{i j}$ parameters are $O(1)$, then $\ell_{i j} \propto \epsilon$.

The resulting transfer function still depends on three distinct modal scales, $\ell_{x x}, \ell_{x y}$ and $\ell_{y y}$, as shown in full in appendix A (A4). However, for algebraic simplicity, we assume that the $\ell_{i j}$ length scales are of similar magnitude and thus let $\ell_{x x}=\ell_{x y}=\ell_{y y}=\ell$, although this assumption can easily be relaxed. The analytical transfer function for the streamwise velocity components expanded in the vicinity of critical layer, is then

$$
\begin{aligned}
& -\frac{1}{\epsilon^{2} R e}\left[\mathrm{i} \frac{\Delta y}{\epsilon}+\epsilon^{2} \ell^{-2}\right] \tilde{R}_{x x}=\left[2 \mathrm{i} k_{x} \bar{r}_{x x}+2 \bar{r}_{x y} \mathrm{~d}-\frac{2\left(\frac{1}{k_{x} \epsilon}\right)\left(\frac{y_{c}}{y}\right)\left(\mathrm{i} k_{x} \bar{r}_{x y}+\bar{r}_{y y} \mathrm{~d}\right)}{\mathrm{i} \frac{\Delta y}{\epsilon}+\epsilon^{2} \ell^{-2}}\right] \tilde{U} \\
& +\left[\bar{r}_{x x, y}-\frac{2\left(\frac{1}{k_{x} \epsilon}\right) \frac{y_{c}}{y}\left(\mathrm{i} k_{x} \bar{r}_{x x}+\bar{r}_{x y} \mathrm{~d}+\bar{r}_{x y, y}\right)}{\mathrm{i} \frac{\Delta y}{\epsilon}+\epsilon^{2} \ell^{-2}}+\frac{\left.2\left(\frac{1}{k_{x} \epsilon}\right)^{2}\left(\frac{y_{c}}{y}\right)^{2}\left(2 \mathrm{i} k_{x} \bar{r}_{x y}+2 \bar{r}_{y y} \mathrm{~d}+\bar{r}_{y y, y}\right)\right] \tilde{V},}{\left[\mathrm{i} \frac{\Delta y}{\epsilon}+\epsilon^{2} \ell^{-2}\right]\left[\mathrm{i} \frac{\Delta y}{\epsilon}+\epsilon^{2} \ell^{-2}\right]}\right]
\end{aligned}
$$

where we observe that the streamwise stress depends on both streamwise and wall-normal large scales, and their spatial derivatives, whereas the experimental measurements typically report the connection between the streamwise components only. To simplify the analysis, we determine the conditions under which the $\tilde{V}$ contribution to the stress can be neglected, by comparing the dominant term in $\tilde{V}$ with the least dominant term in $\tilde{U}$, as elaborated in appendix $\mathrm{A}$, resulting in four conditions

$$
\begin{gathered}
\frac{k_{x} \bar{r}_{x x}}{\bar{r}_{y y, y}} \gtrsim 1, \\
\frac{1}{k_{x}} \frac{\mathrm{d}|\tilde{V}|}{|\tilde{V}|} \lesssim 1, \\
\tan \theta_{U} \lesssim\left|\frac{\bar{r}_{x y}}{\bar{r}_{x x}}\right|, \\
\left|\frac{\tilde{V}}{\tilde{U}}\right| \ll\left(k_{x} \epsilon\right)^{2}\left|\frac{\bar{r}_{x x}}{\bar{r}_{x y}}\right| .
\end{gathered}
$$

Condition (3.10a) indicates that the Reynolds stresses have negligible spatial variation in the vicinity of the critical layer, which is consistent with Townsend's equilibrium layer argument within the logarithmic region of the velocity profile. From the boundary-layer measurements of Jacobi \& McKeon (2013) at $R e_{\tau}=910$, the ratio $\bar{r}_{x x} / \bar{r}_{y y, y} \approx 1.5$ near the critical layer, and thus this is satisfied for all $k_{x} \gtrsim 0.67$.

Condition (3.10b) indicates that the length scale associated with wall-normal changes in $\tilde{V}$ is no smaller than the wavelength of the isolated disturbance, which is already satisfied for most values of $k_{x}$ by the earlier assumption that the peak fluctuating stress occurs near the critical layer.

Condition $(3.10 c)$ depends on the large-scale inclination angle, $\theta_{U}$, and the ratio of the Reynolds stresses. The ratio of the Reynolds streamwise normal stress to other Reynolds stress components, $\left|\bar{r}_{x x} / \bar{r}_{x y}\right|$ or $\left|\bar{r}_{x x} / \bar{r}_{y y}\right|$, is typically $O(10)$, as reported in Fernholz \& 
Finley (1996). And the typical streamwise inclination angle, $\theta_{U} \approx 15^{\circ}$, as reported in Marusic \& Heuer (2007), thus $\tan \theta_{U} \approx 0.27$, satisfying this requirement.

This leaves just the condition $(3.10 d)$ to enforce. Substituting the definition of $\epsilon$ and assuming that, in the logarithmic layer, $\mathrm{d} \bar{u}_{c} \approx\left(\kappa y_{c}\right)^{-1}$ and $y_{c} \approx 3.9 R e^{-1 / 2}$, and again that $\left|\bar{r}_{x x} / \bar{r}_{x y}\right| \sim 10$, yields

$$
\left|\frac{\tilde{V}}{\tilde{U}}\right| \ll 10 k_{x}^{4 / 3} \operatorname{Re}^{-1} .
$$

For $k_{x}=1$ and $R e=910$ (for later comparison with Jacobi \& McKeon (2013)), this means the ratio of wall-normal to streamwise modes must be less than approximately 0.01 , which is almost but not exactly satisfied in numerical calculations of the resolvent modes based on empirical mean velocity profiles, and in the forced modes observed in Jacobi \& McKeon (2017). Formally satisfying the requirement would require large scales of the order of three outer units, $k_{x}=2 \pi / 3$, with $R e \approx 1000$, or larger scales at still smaller Reynolds numbers. Note, however, that this requirement is met by definition for resolvent modes based on the Squire operator rather than the full Orr-Sommerfeld operator, as discussed, e.g. in Dawson \& McKeon (2019). Therefore, we proceed with some caution in neglecting all of the terms pre-multiplying $\tilde{V}$ in (3.9) and presenting the analysis of the transfer function in terms of only the streamwise component, $\tilde{U}$.

The criteria for neglecting the $\tilde{V}$ contribution to the transfer function listed in (3.10) also implicitly relied on an additional assumption, that $\mathrm{d}|\tilde{U}| /|\tilde{U}| \ll\left|\mathrm{d} \phi_{U}\right|$, i.e. that the critical layer occurs very near the maximum in the isolated-scale mode shape. However, this is really the very same assumption we already applied above, with respect to the maximum in the stress profile, where we assumed that $\mathrm{d}\left|\tilde{R}_{x x}\right| /\left|\tilde{R}_{x x}\right| \ll\left|\mathrm{d} \phi_{R}\right|$. The result of this assumption is that the spatial derivatives $\mathrm{d} \tilde{U}$ can be assumed to represent predominantly the variation in large-scale phase, $\mathrm{d} \phi_{U}$ and not magnitude.

The simplified, streamwise transfer function is finally separated into real and imaginary parts, shown in detail in appendix A. A final simplifying assumption is applied to the transfer function: terms of $O\left(k_{x} \epsilon\right)$ compared to the leading-order terms are neglected, to yield

$$
\frac{\tilde{R}_{x x}}{\tilde{U}}=\frac{\epsilon \operatorname{Re}\left(-2 \bar{r}_{x y}-2 \bar{r}_{y y} \frac{\mathrm{d} \phi_{U}}{k_{x}}\right)\left(\frac{y_{c}}{y}\right)}{\left[\left(\frac{\Delta y}{\epsilon}\right)^{2}+\left(\frac{\epsilon}{\ell}\right)^{4}\right]^{2}}\left\{-2\left(\frac{\Delta y}{\epsilon}\right)\left(\frac{\epsilon}{\ell}\right)^{2}+i\left(\left[\left(\frac{\Delta y}{\epsilon}\right)^{2}-\left(\frac{\epsilon}{\ell}\right)^{4}\right]\right)\right\},
$$

where we assume that $d \phi_{U}<0$ to represent downstream inclined structures and $\bar{r}_{x y}<0$ following the usual convention for attached flows. Therefore, $\left(-2 \bar{r}_{x y}-2 \bar{r}_{y y}\left(\mathrm{~d} \phi_{U} / k_{x}\right)\right)>0$. All of the assumptions used in this simplified analysis and the more general treatment in the next section are summarized in table 1 .

The sense of the phase, $\varphi$, is determined by the sign of the imaginary part of the transfer function, which depends on the size of the region of interest, $\Delta y$ surrounding the critical layer as well as the ratio of the critical layer scaling and stress mode scaling, such that

$$
\frac{\Delta y}{\epsilon}<\left(\frac{\epsilon}{\ell}\right)^{2} \Longleftrightarrow \varphi<0 .
$$


General assumptions and restrictions:

$\begin{array}{ll}\text { Upper branch critical layer scaling: } & \epsilon=\left(k_{x} \mathrm{~d} \bar{u}_{c} R e\right)^{-1 / 3} \\ \text { Velocity linearization at critical layer: } & \bar{u}(y) \approx c+\mathrm{d} \bar{u}_{c} \Delta y \\ \text { Stress appears in modal form: } & \mathrm{d}^{2} \tilde{R}_{i j}=R_{2}^{(i j)}(y) \tilde{R}_{i j} \\ \quad \text { Modal length scale: } & \ell_{i j}^{-2}=-\left[R_{2}^{(i j)}(y)-k^{2}\right] \\ \quad \text { Quasi-isotropic modal scales: } & \ell \approx \ell_{x x} \approx \ell_{x y} \approx \ell_{y y} \\ \quad \text { Modal scales similar to critical layer thickness: } & \ell \sim \epsilon\left(\text { equivalently } \alpha_{i j}, \beta_{i j} \sim 1\right) \\ \quad & \frac{\mathrm{d}\left|\tilde{R}_{x x}\right|}{\left|\tilde{R}_{x x}\right|} \ll\left|\mathrm{d} \phi_{R}\right| \\ \text { Neglect } y \text {-dependence in } \ell: & \left|\frac{\tilde{V}}{\tilde{U}}\right| \ll\left(k_{x} \epsilon\right)^{2}\left|\frac{\bar{r}_{x x}}{\bar{r}_{x y}}\right|, \text { see (3.10) } \\ \text { Neglect wall-normal component: } & \frac{\mathrm{d}|\tilde{U}|}{|\tilde{U}|} \ll\left|\mathrm{d} \phi_{U}\right| \\ \text { Evaluate phase near modal maximum: } & \left(k_{x} \epsilon\right) \ll 1\end{array}$

Table 1. Definitions and assumptions made in the analysis of the transfer function. See figure 1 for a schematic view of the quantities involved.

According to the analytical model in (3.8), $\ell$ is the same order of magnitude but nevertheless smaller than $\epsilon$. Therefore, for any finite region of interest $\Delta y \lesssim \epsilon$, the phase is predicted to be negative.

More specifically, we note that the negligible real part of the transfer function (3.12) changes sign across the critical layer, as $\Delta y$ changes sign, and the dominant imaginary part maintains a negative sign everywhere in the vicinity of the critical layer. Therefore, the phase difference between the stress and the isolated large-scale motions is predicted to hover about $-\pi / 2$, crossing the negative imaginary axis, from the fourth quadrant to the third quadrant, as the critical layer is traversed from below. As $y$ approaches $y_{c}$ from below, i.e. $y \rightarrow y_{c}^{-}$, the phase $\varphi$ approaches $-\pi / 2$ from less negative phase angles, i.e. $\varphi \rightarrow-\pi / 2^{+}$. And the reverse is true when approaching the critical layer from above, according to

$$
\left\{\begin{array}{ll}
\varphi \rightarrow-\pi / 2^{+} & \text {as } y \rightarrow y_{c}^{-}, \\
\varphi \rightarrow-\pi / 2^{-} & \text {as } y \rightarrow y_{c}^{+},
\end{array} \quad \text { where } \varphi=\arg \tilde{R}_{x x}-\arg \tilde{U}=\phi_{R}-\phi_{U} .\right.
$$

At the wall, the stress and large scales are identically in phase (since they are both trivially zero) and far from the wall, they have been observed to appear out of phase, likely due to intermittency effects (Mathis et al. 2009a). Therefore, the phase difference at the wall is $\phi(y \rightarrow 0)=0$ and the phase difference near the channel centre (or boundary-layer edge $)$ is expected to approach $\phi(y \rightarrow 1)= \pm \pi$, as illustrated in figure $1(a, c)$. The sign of the phase differences in purely in-phase or out-of-phase orientations is ambiguous and must be established at some intermediate wall location. The present result that, about the critical layer, the phase hovers about $-\pi / 2$, thus establishes the path by which the phase progresses from the wall to the outer flow, through increasingly negative phase differences, such that

$$
\varphi: \quad 0 \rightarrow-\pi / 2 \rightarrow-\pi \quad \text { as } y: \quad 0 \rightarrow y_{c} \rightarrow 1
$$

consistent with previous observations that the small-scale fluctuations lead the large scales in spatial orientation in the flow.

In the limit as the Reynolds number increases, $R e \rightarrow \infty$, the thickness of the viscous critical layer $\epsilon \rightarrow 0$, and thus we can calculate the inviscid limit for the phase relationship 


\section{Interactions between scales in wall turbulence}

between large-scale motions and the fluctuating stresses as

$$
\lim _{\epsilon \rightarrow 0} \frac{\Delta y / \epsilon}{(\epsilon / \ell)^{2}}=\Delta y \lim _{\epsilon \rightarrow 0} \frac{\ell^{2}}{\epsilon^{3}}>1,
$$

assuming fixed, finite $\Delta y$ and asymptotically similar $\epsilon \sim \ell$. Therefore, in the inviscid limit, there is no finite region $\Delta y$ for which the phase is negative, in contrast to the viscous result and experimental measurements. Under this analytical framework, the presence of a finite, viscous critical layer is essential to correctly predicting the experimentally measured sense of phase between scales.

\section{Predictions associated with the very-large-scale motions in the logarithmic region}

Clearly, the transfer functions of (2.8) imply that there are phase relationships between large-scale motions and fluctuating stresses in all three components; we reserve such analysis for future work. However, more advanced modelling using the current understanding of streamwise structure in the log region can be performed; we now exploit our analysis to make predictions regarding the influence of the VLSMs over a finite extent of the logarithmic region.

\subsection{Relationship between the direct correlation coefficient and the amplitude modulation statistic for the very-large-scale motion}

The analysis of $\S 3$ considered the phase relationship between a single isolated scale with Fourier dimensions resembling a VLSM, and stress fluctuations corresponding to all other triadically consistent scales contributing to $\tilde{R}_{x x}$, a relationship associated with the direct correlation coefficient, $\Phi$. The amplitude modulation statistic of (2.14), however, is more broadly defined in terms of a large-scale signal on one side of a (streamwise) wavenumber cutoff and a small-scale signal on the other side, as described in (2.13) and (2.14), and therefore describes a different set of triadically consistent interactions.

Following the analysis of Duvvuri \& McKeon (2015), we write a simple Fourier series decomposition of the streamwise velocity fluctuations

$$
u^{\prime}=\sum_{i=1}^{\infty} \alpha_{i} \sin \left(k_{i} x+\phi_{i}\right)
$$

Here, $k_{1}$ is the largest scale in the signal. The single-scale $(\tilde{u})$ wavenumber is taken to be $k_{L}$ and written as

$$
\tilde{u}=\alpha_{L} \sin \left(k_{L} x+\phi_{L}\right),
$$

and the stress can be composed as the product of the remaining triadic scales as

$$
\tilde{r}_{x x}=\sum_{\substack{\forall m, n \mid \\ k_{n} \mp k_{m}=k_{L}}} \alpha_{m} \sin \left(k_{m} x+\phi_{m}\right) \alpha_{n} \sin \left(k_{n} x+\phi_{n}\right) .
$$

Then, $\Phi$ can be reduced by averaging the covariance between the $\tilde{u}$ and $\tilde{r}_{x x}$ over one wavelength of scale $k_{L}$ to obtain

$$
\Phi=\frac{1}{4 \Omega} \sum_{\substack{\forall m, n \mid \\ k_{n} \mp k_{m}=k_{L}}} \pm \alpha_{L} \alpha_{m} \alpha_{n} \sin \left(\phi_{L}-\left[\phi_{n} \mp \phi_{m}\right]\right),
$$




\section{Jacobi, D. Chung, S. Duvvuri and B.J. McKeon}

where $\Omega=\left\langle(\tilde{u})^{2}\right\rangle^{1 / 2}\left\langle\left(\tilde{r}_{x x}\right)^{2}\right\rangle^{1 / 2}$ is the normalization factor for the covariance $\left\langle\tilde{u} \tilde{r}_{x x}\right\rangle$. It is seen from the above equation that only sets of wavenumbers $k_{m}, k_{n}$ from $u^{\prime}$ that interact in a triadic manner with $\tilde{u}$ (i.e. with $k_{L}$ ) make non-zero contributions to $\Phi$. This is similar to the expression for skewness and $\Psi$ given by Duvvuri \& McKeon (2015).

The amplitude modulation coefficient can be similarly reduced to

$$
\mathcal{R}=\frac{1}{\Omega} \sum_{\substack{\forall L, m, n \mid \\ k_{n}-k_{m}=k_{L} \\ 0<k_{L}<k_{\zeta} \\ k_{m}, k_{n}>k_{\zeta}}} \alpha_{l} \alpha_{m} \alpha_{n} \sin \left(\phi_{L}-\left[\phi_{n}-\phi_{m}\right]\right)
$$

where $\Omega=\sqrt{\left\langle\mathcal{E}_{L}^{2}\right\rangle\left\langle u_{L}^{2}\right\rangle}$ is the normalization factor for the covariance $\left\langle\mathcal{E}_{L} u_{L}\right\rangle$, with $\mathcal{E}_{L}$ denoting the large-scale-pass filtered envelope function. Only triadically consistent interactions with wavenumbers obeying the filtering operation implicit in (2.13), i.e. a subset of $\tilde{r}_{x x}$, make a non-zero contribution to $\mathcal{R}$. Even taking into account the observation that the amplitude modulation coefficient is dominated by the VLSM (Jacobi \& McKeon 2013) such that $u_{L}$ can be reasonably modelled using an isolated VLSM scale, $\tilde{u}, \Phi$ contains scale interactions that are excluded by the filtering process used in $\mathcal{R}$, specifically interactions across the filter such that $k_{n}>k_{l}$ and $k_{m}<k_{l}$.

We argue that the critical layer origin of the correlation phenomena means that these statistics are likely dominated by modes with similar critical layers, since the peak mode amplitudes occur at or near critical layers and the gain, $\left|\tilde{R}_{x x} / \tilde{U}\right|^{2}$, is large at the critical layer. McKeon \& Sharma (2010) have shown that the wall-normal height of the critical layer increases as the streamwise wavenumber decreases, a consequence of the increased convection speed for constant frequency (in general a more subtle influence of the blocking effect of the wall, such that long structures cannot be centred close to the wall). Thus cross-filter contributions to $\tilde{r}_{x x}$ are likely to be small, and most of the triadic contribution to $\tilde{r}_{x x}$ is expected from the small scales. This means that the fluctuating stress term $\tilde{R}_{x x}$ can be interpreted reasonably as a representative of the envelope of small-scale fluctuations in the flow. And, as such, it is reasonable to investigate the similarities between $\Phi$ and $\mathcal{R}$ under the assumption that the influence of the cross-filter triadic interactions is small.

In general terms, the coefficient $\mathcal{R}$ in physical space, with $\mathcal{E}(x, y, z, t)$ and $u_{L}(x, y, z, t)$ is defined as

$$
\mathcal{R}(y)=\frac{\iiint \mathcal{E}^{*} u_{L} \mathrm{~d} x \mathrm{~d} z \mathrm{~d} t}{\left(\iiint \mathcal{E}^{*} \mathcal{E} \mathrm{d} x \mathrm{~d} z \mathrm{~d} t\right)^{1 / 2}\left(\iiint u_{L}^{*} u_{L} \mathrm{~d} x \mathrm{~d} z \mathrm{~d} t\right)^{1 / 2}} .
$$

The covariance, represented by $\mathcal{R}$, can also be expressed as the real part of the complex cross-spectrum in Fourier space (i.e. the cospectrum). Writing the Fourier transformed velocity signals as $\hat{u}\left(k_{x}, k_{z}, \omega\right)=\mathcal{F}[u(x, z, t)]$ yields, with the application of the cross-correlation theorem (generalized Wiener-Khinchin theorem)

$$
\mathcal{R}(y)=\mathbb{R} e\left\{\frac{\iiint \hat{\mathcal{E}}^{*} \hat{u}_{L} \mathrm{~d} k_{x} \mathrm{~d} k_{z} \mathrm{~d} \omega}{\left(\iiint \hat{\mathcal{E}}^{*} \hat{\mathcal{E}} \mathrm{d} k_{x} \mathrm{~d} k_{z} \mathrm{~d} \omega\right)^{1 / 2}\left(\iiint \hat{u}_{L}^{*} \hat{u}_{L} \mathrm{~d} k_{x} \mathrm{~d} k_{z} \mathrm{~d} \omega\right)^{1 / 2}}\right\} .
$$

The power spectral densities for the velocity signals are denoted $\psi_{L}=\hat{u}_{L}^{*}{\widehat{u_{L}}}$ and $\psi_{S S}=$ $\hat{\mathcal{E}}^{*} \hat{\mathcal{E}}$, and $\hat{\mathcal{E}}=|\hat{\mathcal{E}}| \mathrm{e}^{\mathrm{i} \phi_{S}}$ and $\hat{u}_{L}=\left|\hat{u}_{L}\right| \mathrm{e}^{\mathrm{i} \phi_{L}}$, where the phases $\phi_{L}$ and $\phi_{S}$ are wavenumber 


\section{Interactions between scales in wall turbulence}

and frequency dependent. The signals in spectral space are related by

$$
\hat{\mathcal{E}} \hat{u}_{L}^{*}=\hat{\mathcal{E}}^{*} \hat{u}_{L} \exp \left(2 i\left(\phi_{S}-\phi_{L}\right)\right)
$$

thus yielding

$$
\mathcal{R}(y)=\mathbb{R} e\left\{\frac{\iiint \psi_{S S}^{1 / 2} \psi_{L L}^{1 / 2} \mathrm{e}^{-\mathrm{i}\left(\phi_{S}-\phi_{L}\right)} \mathrm{d} k_{x} \mathrm{~d} k_{z} \mathrm{~d} \omega}{\left(\iiint \psi_{S S} \mathrm{~d} k_{x} \mathrm{~d} k_{z} \mathrm{~d} \omega\right)^{1 / 2}\left(\iiint \psi_{L L} \mathrm{~d} k_{x} \mathrm{~d} k_{z} \mathrm{~d} \omega\right)^{1 / 2}}\right\},
$$

where $\varphi=\phi_{S}-\phi_{L}$ and $\varphi \equiv \varphi\left(k_{x}, k_{z}, \omega\right)$. Taking the real part, we note that the sign of the phase difference, $\varphi$, is lost due to the symmetry of the cosine function

$$
\mathcal{R}(y)=\frac{\iiint \psi_{S S}^{1 / 2} \psi_{L L}^{1 / 2} \cos \varphi \mathrm{d} k_{x} \mathrm{~d} k_{z} \mathrm{~d} \omega}{\left(\iiint \psi_{S S} \mathrm{~d} k_{x} \mathrm{~d} k_{z} \mathrm{~d} \omega\right)^{1 / 2}\left(\iiint \psi_{L L} \mathrm{~d} k_{x} \mathrm{~d} k_{z} \mathrm{~d} \omega\right)^{1 / 2}} .
$$

Consider a large-scale signal that can be reasonably modelled by a VLSM with wavenumber and frequency given by $\left(k_{x}, k_{z}, \omega\right)=\left(k_{L}, a_{L} k_{L}, c_{L} k_{L}\right)$. Imposing a narrow bandpass filter precisely at this VLSM to reflect the dominance of the very large scale, these spectra are idealized as

$$
\left\{\psi_{S S}, \psi_{L L}\right\} \propto \delta\left(k_{x}-k_{L}\right) \delta\left(k_{z}-a_{L} k_{L}\right) \delta\left(\omega-c_{L} k_{L}\right),
$$

and substituting the spectra into (4.10) yields

$$
\mathcal{R}(y) \approx \cos \left[\varphi\left(k_{L}, a k_{L}, c_{L} k_{L}\right)\right] \approx \Phi\left(k_{L}, a_{L} k_{L}, c_{L} k_{L}\right),
$$

where now $\varphi=\phi_{R}-\phi_{L}$ and $\Phi$ is the direct correlation coefficient evaluated at the VLSM. Thus the value of $\mathcal{R}$ can be interpreted as the cosine of the phase separating the scales, just like the function $\Phi$.

But, as noted above, the correlation coefficients, $\mathcal{R}$ and $\Phi$, obscure the signed sense of this phase difference due to their symmetry. However, the full spatial (or temporal) cross-correlation function, $r(\Delta x, y)$ can be plotted instead of the correlation coefficient, in order to identify the correct sense of the phase lag (e.g. Chung \& McKeon 2010; Hutchins et al. 2011; Jacobi \& McKeon 2013). The spatial cross-correlation function, in the streamwise direction, is defined as

$$
r(\Delta x, y)=\frac{\iiint \mathcal{E}^{*}(x, y, z, t) u_{L}(x+\Delta x, y, z, t) \mathrm{d} x \mathrm{~d} z \mathrm{~d} t}{\left(\iiint \mathcal{E}^{*} \mathcal{E} \mathrm{d} x \mathrm{~d} z \mathrm{~d} t\right)^{1 / 2}\left(\iiint u_{L}^{*} u_{L} \mathrm{~d} x \mathrm{~d} z \mathrm{~d} t\right)^{1 / 2}},
$$

such that $\Delta x>0$ indicates that the large scales lead the small scales, spatially. (If defined temporally, then $\Delta t>0$ would small scales lead large scales, spatially.) The correlation coefficient is related to the cross-correlation function as: $\mathcal{R}(y)=r(0, y)$, and also by $\mathcal{R}(y)=\cos (\varphi)$ where the phase $\varphi$ can be related to the spatial or temporal lag via $\varphi \propto\left(\left.\Delta x\right|_{r=r_{\max }}\right) / \lambda_{\zeta}$ where $\lambda_{\zeta}$ is the spatial filter size used to separate between large and small scales and the proportionality is fixed such that $\mathcal{R}=0$ corresponds to $|\varphi|=\pi / 2$. The three different representations of the phase are shown in figure 2 .

According to the analysis of the preceding sections, $\varphi=-\pi / 2$, i.e. $\mathcal{R}=0$, in the vicinity of $y_{c}$, the critical layer. Further, the phase lead increases with height, i.e. the $\tilde{R}_{x x}$ structure leads the $\tilde{U}$ structure and a higher stress region can be found downstream of a higher velocity region, and crosses $-\pi / 2$ at $y_{c}$ in agreement with the observations of the VLSM and consistent with the measurements shown in figure 2. This is likely the 
(a)

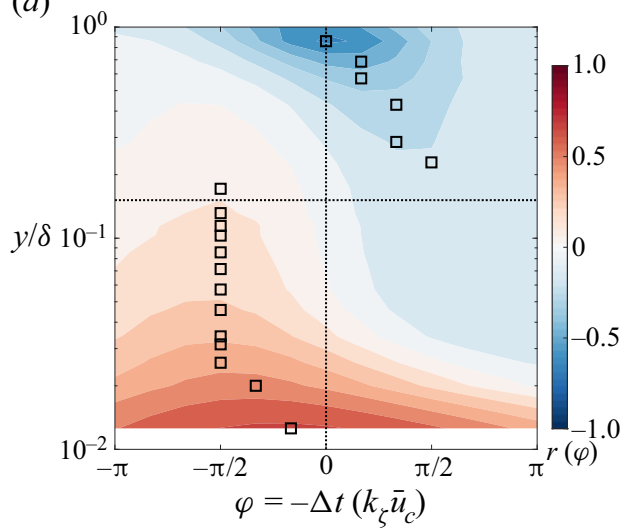

(b)

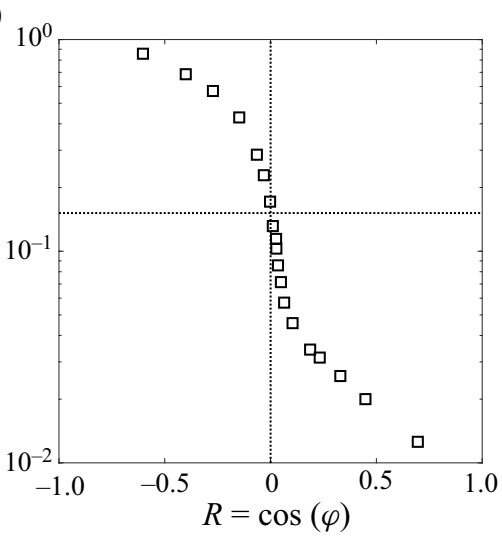

(c)

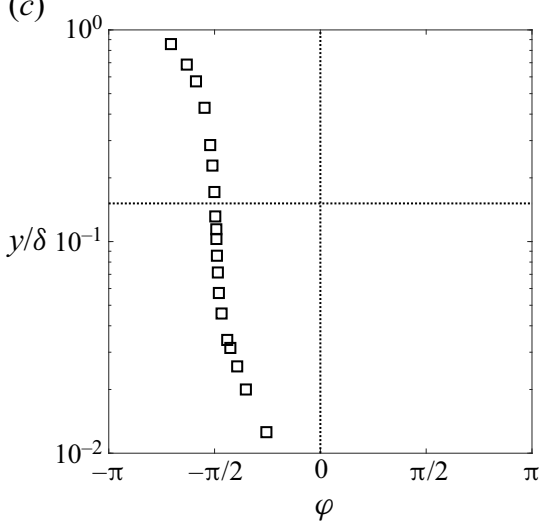

Figure 2. (a) The correlation coefficient with symbols from Jacobi \& McKeon (2013) measured at $R e_{\tau}=$ 910 using a temporal filter cutoff between large and small scales of $1 \delta / U_{\infty}$. (b) Map of the temporal cross-correlation function, $r(\Delta t, y)$ with the peaks marked, transformed into the phase coordinate, $r(\varphi, y)$ by scaling via a wavenumber very close to the filter wavenumber, $k_{\zeta}$. (The precise wavenumber for the transformation is selected such that the peak location corresponds to $\cos ^{-1}(R)$.) The correlation coefficient $R$ corresponds to the value of $r(\varphi=0)$ and also, equivalently, to the cosine of the phase, $\varphi$ evaluated at the marked peaks. (c) The phase, evaluated from the transformed time-lag information in $(b)$, where the sense of the phase is clearly negative.

origin of earlier hypotheses (Chung \& McKeon 2010; Hutchins et al. 2011) concerning the dependence of the amplitude modulation coefficient on the streamwise gradient of $\tilde{U}$. Note that the shape of $\mathcal{R}$ could change if a weighted integral were to be taken over the full range of large scales as opposed to the single dominant mode but we expect these to be higher-order corrections to (4.12).

\subsection{Structure angles for the stress fluctuation and large-scale velocity}

The transfer function analysis of $\S 3$ identified the sense of the phase between the stress fluctuations and the isolated scale through the properties of the critical layer, a detail that is not captured in the amplitude modulation coefficient due to the symmetry of the cosine function. In this section, we use the predicted relative phase to reconstruct the physical relationships between large- and small-scale activity. 


\section{Interactions between scales in wall turbulence}

A local isolated-scale structure inclination angle, $\theta_{U}$, and an analogous stress-fluctuation structure inclination angle, $\theta_{R}$, can be introduced, and related using (2.10)

$$
-\frac{k_{L}}{\tan \theta_{R}} \equiv \frac{\mathrm{d} \arg \tilde{R}_{x x}}{\mathrm{~d} y}=\frac{\mathrm{d} \varphi}{\mathrm{d} y}+\frac{\mathrm{d} \arg \tilde{U}}{\mathrm{~d} y}=\frac{\mathrm{d} \varphi}{\mathrm{d} y}-\frac{k_{L}}{\tan \theta_{U}} .
$$

The phase shift in the vicinity of the critical layer can be extracted from the ratio of the real and imaginary parts of (3.12) and written as

$$
\varphi \approx \arctan \left[\frac{\left(\frac{\Delta y}{\epsilon}\right)^{2}-\left(\frac{\epsilon}{\ell}\right)^{4}}{-2\left(\frac{\Delta y}{\epsilon}\right)\left(\frac{\epsilon}{\ell}\right)^{2}}\right],
$$

where the branch of the inverse tangent is taken to be $[0,-\pi)$, consistent with the result that the phase at the critical layer hovers around $-\pi / 2$. Then differentiating with respect to $y$ and evaluating at the critical layer, and taking the limit for $\Delta y / \epsilon \ll 1$, yields

$$
\begin{aligned}
\frac{\mathrm{d} \varphi}{\mathrm{d} y} & =-\frac{1}{\epsilon} \frac{2\left(\frac{\ell}{\epsilon}\right)^{2}}{1+\left(\frac{\ell}{\epsilon}\right)^{4}\left(\frac{\Delta y}{\epsilon}\right)^{2}} \approx-2\left(\frac{\ell}{\epsilon}\right)^{2} \epsilon^{-1} \\
& \approx-2\left(\frac{\ell}{\epsilon}\right)^{2}\left(k_{L} \mathrm{~d} \bar{u}_{c} R e\right)^{1 / 3} .
\end{aligned}
$$

Together with (4.14), (4.17) says that the relative inclination between the $\tilde{U}$ and $\tilde{R}_{x x}$ structures at $y_{c}$ depends on the thickness of the critical layer, to leading order. Combining (4.14) and (4.17), and assuming small angles for $\theta_{U}$ and $\theta_{S}$, we obtain, to leading order,

$$
\left.\left(\theta_{R}-\theta_{U}\right)\right|_{c} \approx-\theta_{U}\left(\frac{2\left(\frac{\ell}{\epsilon}\right)^{2} \frac{\theta_{U}}{k_{L} \epsilon}}{1+2\left(\frac{\ell}{\epsilon}\right)^{2} \frac{\theta_{U}}{k_{L} \epsilon}}\right)<0 .
$$

That is, the $\tilde{R}_{x x}$ structure is more inclined to the wall than the $\tilde{U}$ structure at $y_{c}$, the difference controlled by $R e, k_{L}$ and $\mathrm{d} \bar{u}_{c}$. These qualitative predictions of the scale interaction are corroborated by the boundary-layer experiments of Hutchins et al. (2011) and Jacobi \& McKeon (2013), in which the small-scale activity leads the very-large-scale motion, but differ from those reported in Bandyopadhyay \& Hussain (1984) (figures 2, 8 and 14a). Chung \& McKeon (2010) and Jacobi \& McKeon (2013) previously sketched such a relationship between effective structure angles of the VLSM and small-scale stresses in the context of the amplitude modulation coefficient. The present analysis suggests a similar sketch of the relative orientation of large- and small-scale modes, as shown in figure 3 .

\subsection{The amplitude modulation statistic in the log region}

Further progress in understanding the variation of the amplitude modulation coefficient can be made by applying the results of $\S 3$ to describe the phase over the full region $\Delta y$ 

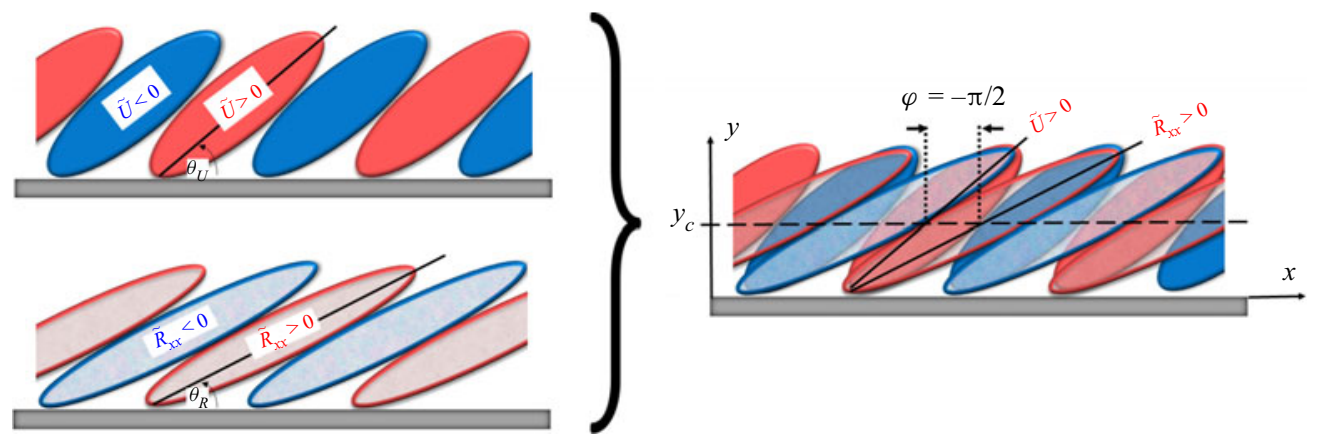

Figure 3. A schematic representation of the relative orientation of the large-scale motions and the envelope of the small-scale stresses as indicated by the viscous analysis, showing that the positive small-scale signal $R_{x x}$ leads the large, $\tilde{U}$, and thus the relative inclination of the small scales is steeper than that of the large scales (after Chung \& McKeon 2010) according to (4.18). Near the wall, both scales are in phase, and away from the wall they are exactly out of phase, as suggested in (3.15).
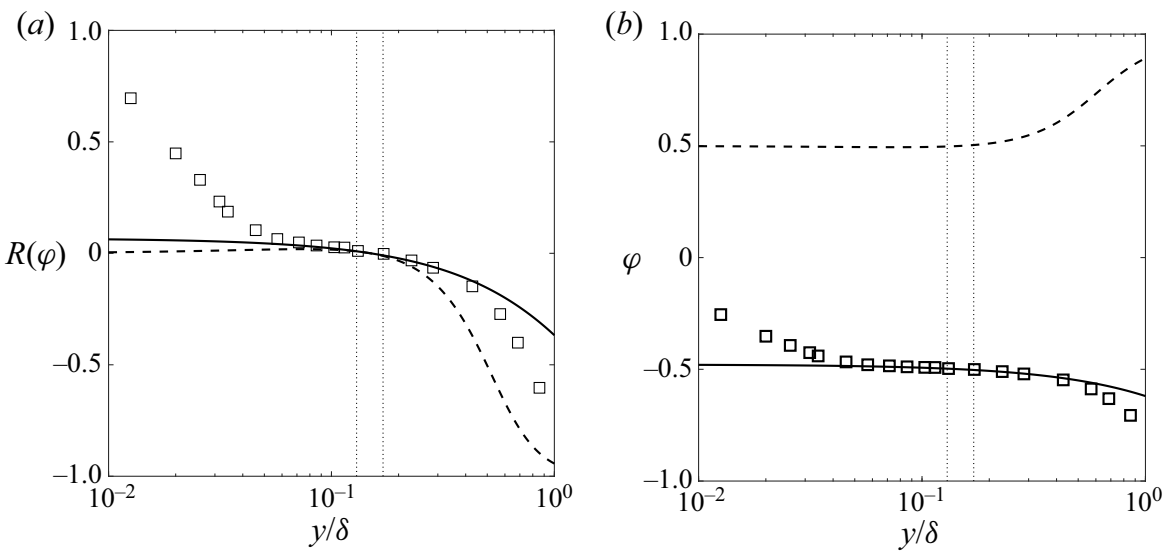

Figure 4. (a) The correlation coefficient, as shown in figure 2. Here, the solid line is the viscous model from (4.15) with $(\ell / \epsilon, B)=(0.1,15)$, where the actual values of the Reynolds stress functions were estimated, using $\theta_{U} \approx 15^{\circ}$, as $B\left(y_{c}\right) \approx 13$. The dashed line is the inviscid model from (A15) with $(A, B) \approx(10,20)$. Note that both models capture the trend in $R$ reasonably well, in an $\epsilon$-size region about the vicinity of the critical layer, marked by the dotted lines. $(b)$ The phase, evaluated from the time-lag information in figure 2 . Here, the solid line is the viscous prediction; the dashed line is the inviscid prediction. Note that the inviscid model predicts the incorrect sense of phase.

about the critical point $y_{c}$, and even beyond. We can illustrate this by plotting $\mathcal{R}=\cos \varphi$ with $\varphi$ given by (4.15). As before, there are two free parameters to fit to this phase model: the length scale associated with the fluctuating stress, $\ell$, and the function $B(y)$ (defined in (A10)) that represents the magnitude of the average Reynolds stresses. We now fit the model (4.15) to the $R e_{\tau}=910$ boundary-layer data from Jacobi \& McKeon (2013), see figure 4 . The model demonstrates reasonable agreement for a range of $y$ beyond the immediate vicinity of the critical layer. The zero crossing at $y=y_{c}$ is captured, confirming the hypothesized relationship between the outer layer peak, this zero crossing (Mathis et al. 2009a), and the VLSM critical layer. However, the model fails to capture the subtle peak or plateau observed in the vicinity of the critical layer itself.

Performing the same phase analysis on the inviscid phase difference, detailed in appendix A yields a very similar prediction for $\mathcal{R}$, shown in the dashed line in figure 4(a). 


\section{Interactions between scales in wall turbulence}

But, importantly, the inviscid model fails to predict the correct sense of phase, in figure $4(b)$, as explained above.

The wavenumber corresponding to the large-scale activity, $k_{L} \delta$, appears to be independent of $\operatorname{Re}_{\tau}$ (see Mathis et al. 2009a). If the same holds approximately for $\theta_{U}$, as appears to be the case in the results of Marusic \& Heuer (2007) who found an inclination angle of approximately $15^{\circ}$ over a range of Reynolds number spanning laboratory to atmospheric boundary-layer conditions, then $\mathcal{R}_{\max }$ primarily depends on $y_{c}$, a function of $R e_{\tau}$, but also on the choice of filter cutoff, $k_{\zeta}$, in terms of the restriction it puts onto allowed triadic interactions (see $\S 4.1$ ). The sensitivity to $k_{\zeta}$ and $R e_{\tau}$ of the peak, but not the zero-crossing height is reported by Mathis et al. (2009a). In more recent work, McKeon $\&$ Sharma (2010) indicated that $y_{c}$ for the VLSM should scale with $R e^{1 / 2}$. Note that if, as reported, $\mathcal{R}_{\max }$ were to increase with $\operatorname{Re}_{\tau}$ then $\beta_{c} /\left(y_{c}\right)$ must decrease accordingly.

\section{Concluding remarks}

The dynamical equations for an isolated scale, containing a single streamwise/spanwise wavenumber and frequency $\left(k_{x}, k_{z}, \omega\right)$, and the associated stress fluctuations at the same scale were reformulated in terms of a transfer function relationship, in line with the input-output approach of the resolvent analysis of McKeon \& Sharma (2010). In this work, we have investigated the transfer function governing the well-studied relationship between the streamwise stress fluctuations and the large-scale streamwise velocity.

After some simplifying assumptions, the transfer function clearly identifies the role of the viscous critical layer in the amplitude and phase relationship between the single scale and stress components. Specifically, we assume that the unclosed terms on the right-hand side of (2.4) give rise to stress fluctuations that are uncorrelated with the velocity at the isolated scale. A modal form for the velocity and, through quadratic nonlinear interaction, the stress, is assumed in order to invert the matrix; this is consistent with the recent analytical work of Dawson \& McKeon (2019). Finally, the contribution of the wall-normal large-scale velocity component may only be formally neglected under a certain set of conditions. These simplifying assumptions resulted in a predicted phase relationship in the vicinity of the large-scale critical layer consistent with experimental observations. A full discretization of (2.8) may still be desirable, particularly for investigating the phase relationships away from the critical layer itself, but the present analysis has shown that near the critical layer, the phase does not depend significantly on the precise form of the mean Reynolds stress profiles, beyond certain order-of-magnitude approximations. We note that inclusion of viscosity is, however, vital: making an inviscid assumption leads to the incorrect sense of the phase lead/lag.

Representing the VLSM via a single scale, consistent with the observations of Jacobi \& McKeon (2013) that the VLSMs dominate the phase relationship between large-scale velocity and the envelope of small-scale fluctuations, a prediction of the phase relationship between the streamwise small-scale stress and VLSM velocity was obtained. This prediction was shown to be in good agreement with experimental observations, as well as the quasi-steady model of Chernyshenko et al. (2012), and was subsequently used to obtain insight into the origin of structure angles for the large and small scales, and the form of the amplitude modulation statistic in the logarithmic region. Consistent with experiments, the model predicts that the zero-crossing height of the amplitude modulation statistic coincides with the wall-normal location of the very-large-scale peak in the one-dimensional premultiplied spectrum of streamwise velocity fluctuations, the critical layer location for the very-large-scale motion. For the VLSM, the transfer function between 


\section{Jacobi, D. Chung, S. Duvvuri and B.J. McKeon}

streamwise velocity and streamwise stress is closely related to the direct correlation coefficient and to the amplitude modulation statistic.

The relationship between streamwise velocity and streamwise stress has been explored here, with connections to an expansion of the empirical predictive model proposed by Marusic et al. (2010), which effectively leverages phase information integrated into the behaviour of the small scales through an off-wall measurement of the large-scale streamwise velocity. However, we note that the transfer functions at other scales, and between other velocity components, have equivalent (if mathematically more complex) relationships described in full by (2.8). In addition, Luhar et al. (2014) extended the resolvent analysis of McKeon \& Sharma (2010) to explicitly include the pressure variation (corresponding in essence to the 'fast' pressure identified in the Poisson equation for the pressure fluctuations) and postulated that pressure fluctuations must experience a similar amplitude modulation effect to the velocity. Thus the phase relationships introduced herein can be identified as a fundamental restriction on interactions between triadically consistent scales, with strong implications for modelling, and particularly for the small-scale stress fluctuations which could reasonably be incorporated into a subgrid-scale model for large eddy simulation.

Moreover, we note that gradients of $\tilde{R}_{i j}$ constitute the forcing term, $f_{i}$, in $(2.3 a)$ for the isolated scale, and thus are identically the nonlinear forcing terms in the resolvent analysis of McKeon \& Sharma (2010). As such, information on the relationships between $\tilde{U}_{i}$ and $\tilde{R}_{i j}$ provide the potential for simplification of the resolvent analysis itself with regards to identifying triadic interactions that sustain particular isolated scales. This is a topic of ongoing study.

Acknowledgements. This research was carried out over a period of years. We gratefully acknowledge support from (D.C.) a National Aeronautics and Space Administration contract at the Jet Propulsion Laboratory, California Institute of Technology, and from (B.J.M.) the Air Force Office of Scientific Research (grants FA9550-09-1-0701 and FA9550-12-1-0469) and Office of Naval Research (grants N00014-17-1-2307 and N00014-17-1-3022). We also thank the anonymous referees for insightful suggestions.

Declaration of interests. The authors report no conflict of interest.
Author ORCIDs.
(1) Ian Jacobi http://orcid.org/0000-0001-7377-8292;
Daniel Chung http://orcid.org/0000-0003-3732-364X;
Subrahmanyam Duvvuri http://orcid.org/0000-0001-8082-1658;
Deverley J. McKeon http://orcid.org/0000-0003-4220-1583.

\section{Appendix A. Three-dimensional transfer function}

\section{A.1. Viscous transfer function}

To obtain the transfer function between $\tilde{R}_{x x}$ and the isolated large scales, we solve (3.5) with the assumption that the $\tilde{R}_{i j}$ components can be represented in modal form, according to the analytical model in (3.3). We define that modal form explicitly, such that the second derivative of the mode is equal to the mode itself scaled by a function $R_{2}^{(i j)}(y)$, according to

$$
\mathrm{d}^{2} \tilde{R}_{i j}=R_{2}^{(i j)}(y) \tilde{R}_{i j}
$$




\section{Interactions between scales in wall turbulence}

which allows us to write the viscous terms of the governing equation $-\left(\mathrm{d}^{2}-k^{2}\right) \tilde{R}_{i j}$ as

$$
-\left(\mathrm{d}^{2}-k^{2}\right) \tilde{R}_{i j}=\underbrace{-\left[R_{2}^{(i j)}(y)-k^{2}\right]}_{\ell_{i j}^{-2}} \tilde{R}_{i j},
$$

where the $\ell_{i j}$ length scale represents the local curvature of the stress modes, as defined in the main body.

The $\tilde{R}_{x x}$ component of (3.5) is then found by inverting the $\boldsymbol{A}$ matrix to obtain

$$
\begin{aligned}
- & {\left[\mathrm{i}\left(-\omega+k_{x} \bar{u}\right)+\operatorname{Re}^{-1}\left(k^{2}-R_{2}^{(x x)}\right)\right] \tilde{R}_{x x} } \\
= & {\left[2 \mathrm{i} k_{x} \bar{r}_{x x}+2 \bar{r}_{x y} \mathrm{~d}-\frac{2 \mathrm{i} \gamma\left(-\omega+k_{x} \bar{u}\right)\left(\mathrm{i} k_{x} \bar{r}_{x y}+\bar{r}_{y y} \mathrm{~d}\right)}{\operatorname{Re}^{-1}\left(k^{2}-R_{2}^{(x y)}\right)+\mathrm{i}\left(-\omega+k_{x} \bar{u}\right)}\right] \tilde{U} } \\
& +\left[\bar{r}_{x x, y}-\frac{2 \mathrm{i} \gamma\left(-\omega+k_{x} \bar{u}\right)\left(\mathrm{i} k_{x} \bar{r}_{x x}+\bar{r}_{x y} \mathrm{~d}+\bar{r}_{x y, y}\right)}{\operatorname{Re}^{-1}\left(k^{2}-R_{2}^{(x y)}\right)+\mathrm{i}\left(-\omega+k_{x} \bar{u}\right)}\right. \\
& \left.-\frac{2 \gamma^{2}\left(-\omega+k_{x} \bar{u}\right)^{2}\left(2 \mathrm{i} k_{x} \bar{r}_{x y}+2 \bar{r}_{y y} \mathrm{~d}+\bar{r}_{y y, y}\right)}{\left[\operatorname{Re}^{-1}\left(k^{2}-R_{2}^{(x y)}\right)+\mathrm{i}\left(-\omega+k_{x} \bar{u}\right)\right]\left[\operatorname{Re}^{-1}\left(k^{2}-R_{2}^{(y y)}\right)+\mathrm{i}\left(-\omega+k_{x} \bar{u}\right)\right]}\right] \tilde{V} .
\end{aligned}
$$

This general transfer function can then be expanded in a narrow region $\Delta y$ about $y_{c}$, to obtain

$$
\begin{aligned}
& -\frac{1}{\epsilon^{2} \operatorname{Re}}\left[\mathrm{i} \frac{\Delta y}{\epsilon}+\epsilon^{2} \ell_{x x}^{-2}\right] \tilde{R}_{x x}=\left[2 \mathrm{i} k_{x} \bar{r}_{x x}+2 \bar{r}_{x y} \mathrm{~d}-\frac{2\left(\frac{1}{k_{x} \epsilon}\right)\left(\frac{y_{c}}{y}\right)\left(\mathrm{i} k_{x} \bar{r}_{x y}+\bar{r}_{y y} \mathrm{~d}\right)}{\mathrm{i} \frac{\Delta y}{\epsilon}+\epsilon^{2} \ell_{x y}^{-2}}\right] \tilde{U} \\
& +\left[\bar{r}_{x x, y}-\frac{2\left(\frac{1}{k_{x} \epsilon}\right) \frac{y_{c}}{y}\left(\mathrm{i} k_{x} \bar{r}_{x x}+\bar{r}_{x y} \mathrm{~d}+\bar{r}_{x y, y}\right)}{\mathrm{i} \frac{\Delta y}{\epsilon}+\epsilon^{2} \ell_{x y}^{-2}}\right. \\
& \left.+\frac{2\left(\frac{1}{k_{x} \epsilon}\right)^{2}\left(\frac{y_{c}}{y}\right)^{2}\left(2 \mathrm{i} k_{x} \bar{r}_{x y}+2 \bar{r}_{y y} \mathrm{~d}+\bar{r}_{y y, y}\right)}{\left[\mathrm{i} \frac{\Delta y}{\epsilon}+\epsilon^{2} \ell_{x y}^{-2}\right]\left[\mathrm{i} \frac{\Delta y}{\epsilon}+\epsilon^{2} \ell_{y y}^{-2}\right]}\right] \tilde{V}
\end{aligned}
$$

and finally applying the assumption that the $\ell_{i j}$ scales are quasi-isotropic yields (3.9).

\section{A.2. Simplifying the transfer function}

The transfer function for the streamwise component of the stress, $\tilde{R}_{x x}$, in (3.9), expanded about the critical layer, can be simplified according to two additional assumptions: (i) negligible contributions to $\tilde{R}_{x x}$ from the $\tilde{V}$ component and (ii) negligible spatial variation in the amplitude of the $\tilde{U}$ component. Both of these simplifying assumptions are consistent with the typical mode shapes of resolvent modes, in which $\tilde{U}$ exhibits 


\section{Jacobi, D. Chung, S. Duvvuri and B.J. McKeon}

its maximum magnitude near the critical layer, and the magnitude of $\tilde{V}$ is everywhere significantly less than that of $\tilde{U}$.

First, we analyse under what conditions $\tilde{V}$ can be neglected. Among the contributions to the $\tilde{V}$ term, the largest magnitude term is $O\left[\left(k_{x} \epsilon\right)^{-2}\right]$, assuming the $\ell_{i j} \sim \epsilon$ and taking the limit as $\Delta y / \epsilon \ll 1$. Similarly, among the contributions to the $\tilde{U}$ term, the $k_{x} \bar{r}_{x x}$ and $\bar{r}_{x y} d$ terms are the smallest. Therefore, to neglect $\tilde{V}$, we must establish that the smallest terms in $\tilde{U}$ still dominate the largest in $\tilde{V}$

$$
\left[2 \mathrm{i} k_{x} \bar{r}_{x x}+2 \bar{r}_{x y} \mathrm{~d}\right] \tilde{U} \gg 2\left(\frac{1}{k_{x} \epsilon}\right)^{2}\left(\frac{y_{c}}{y}\right)^{2}\left[\left(2 \mathrm{i} k_{x} \bar{r}_{x y}+2 \bar{r}_{y y} \mathrm{~d}+\bar{r}_{y y, y}\right)\right] \tilde{V},
$$

which can be expanded as

$$
\begin{aligned}
& {\left[2 \mathrm{i} k_{x} \bar{r}_{x x}+2 \bar{r}_{x y}\left(\frac{\mathrm{d}|\tilde{U}|}{|\tilde{U}|}+\mathrm{id} \phi_{U}\right)\right] \tilde{U}} \\
& \gg 2\left(\frac{1}{k_{x} \epsilon}\right)^{2}\left(\frac{y_{c}}{y}\right)^{2}\left[\left(2 \mathrm{i} k_{x} \bar{r}_{x y}+2 \bar{r}_{y y}\left(\frac{\mathrm{d}|\tilde{V}|}{|\tilde{V}|}+\mathrm{id} \phi_{V}\right)+\bar{r}_{y y, y}\right)\right] \tilde{V} .
\end{aligned}
$$

We already assumed earlier that the critical layer and modal large-scale maximum are very close, $\left|y_{c}-y_{m}\right| / \epsilon \ll \alpha_{U} /\left(2 \beta_{U}\right)$, making use of the analytical formulation in (3.2). This is equivalent to assuming that the $\mathrm{d}|\tilde{U}| /|\tilde{U}| \ll\left|\mathrm{d} \phi_{U}\right|$.

This condition can be simplified to four requirements, making use of the relationship that $\mathrm{d} \phi_{U} \approx-k_{x} / \tan \theta_{U}$ for large-scale stream inclination angle $\theta_{U}$

$$
\left|\frac{\tilde{V}}{\tilde{U}}\right| \ll\left(k_{x} \epsilon\right)^{2}\left|\frac{\bar{r}_{x x}}{\bar{r}_{x y}}\right|, \quad \frac{k_{x} \bar{r}_{x x}}{\bar{r}_{y y, y}} \gtrsim 1, \quad \frac{1}{k_{x}} \frac{\mathrm{d}|\tilde{V}|}{|\tilde{V}|} \lesssim 1, \quad \tan \theta_{U} \lesssim\left|\frac{\bar{r}_{x y}}{\bar{r}_{x x}}\right|,
$$

where the condition on the variation in the amplitude of $\tilde{V}$ can also be written in terms of the semi-analytical model described below (3.2)

$$
\frac{1}{k_{x}} \frac{\mathrm{d}|\tilde{V}|}{|\tilde{V}|} \lesssim 1 \Longleftrightarrow \frac{y_{c}-y_{m, V}}{\epsilon} \lesssim\left(\frac{1}{\sqrt{2 \beta_{V}}}\right)\left(\frac{k_{x} \epsilon}{\sqrt{2 \beta_{V}}}\right)
$$

and thus we observe that the condition on neglecting $\tilde{V}$ is automatically satisfied by the earlier assumption that $\ell_{y y}>0$ independent of $y$.

Neglecting $\tilde{V}$ yields

$$
-\frac{1}{\epsilon^{2} \operatorname{Re}}\left[\mathrm{i} \frac{\Delta y}{\epsilon}+\epsilon^{2} \ell_{x x}^{-2}\right] \tilde{R}_{x x}=\left[2 \mathrm{i} k_{x} \bar{r}_{x x}+2 \bar{r}_{x y} \mathrm{~d}-\frac{2\left(\frac{1}{k_{x} \epsilon}\right)\left(\frac{y_{c}}{y}\right)\left(\mathrm{i} k_{x} \bar{r}_{x y}+\bar{r}_{y y} \mathrm{~d}\right)}{\mathrm{i} \frac{\Delta y}{\epsilon}+\epsilon^{2} \ell_{x y}^{-2}}\right] \tilde{U} .
$$

Expanding the terms involving $\mathrm{d} \tilde{U}$, dividing through by $\tilde{U}$ and applying the above assumption on the location of the modal maximum, which is equivalent to assuming that 
$\mathrm{d}|\tilde{U}| /|\tilde{U}| \ll\left|\mathrm{d} \phi_{U}\right|$, yields

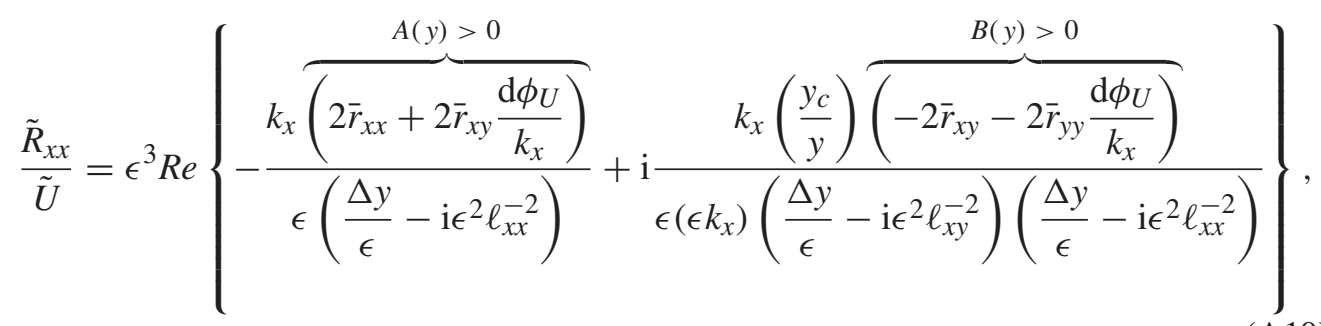

where the combination of Reynolds stress and large-scale phase terms are represented by real, positive functions $A(y), B(y)$. Finally, assuming $\ell_{x x}=\ell_{x y}=\ell_{y y}=\ell$ and separating the real and imaginary parts then yields

$$
\begin{aligned}
\frac{\tilde{R}_{x x}}{\tilde{U}}= & \frac{\epsilon R e}{\left[\left(\frac{\Delta y}{\epsilon}\right)^{2}+\left(\frac{\epsilon}{\ell}\right)^{4}\right]^{2}} \\
& \times\left\{-\left(\frac{\Delta y}{\epsilon}\right)\left(2 B(y)\left(\frac{y_{c}}{y}\right)\left(\frac{\epsilon}{\ell}\right)^{2}+A(y)\left(k_{x} \epsilon\right)\left[\left(\frac{\Delta y}{\epsilon}\right)^{2}+\left(\frac{\epsilon}{\ell}\right)^{4}\right]\right)\right. \\
& \left.+\mathrm{i}\left(-A(y)\left(k_{x} \epsilon\right)\left(\frac{\epsilon}{\ell}\right)^{2}\left[\left(\frac{\Delta y}{\epsilon}\right)^{2}+\left(\frac{\epsilon}{\ell}\right)^{4}\right]+B(y)\left(\frac{y_{c}}{y}\right)\left[\left(\frac{\Delta y}{\epsilon}\right)^{2}-\left(\frac{\epsilon}{\ell}\right)^{4}\right]\right)\right\} .
\end{aligned}
$$

Neglecting small $\left(\epsilon k_{x}\right) \ll 1$ yields

$$
\frac{\tilde{R}_{x x}}{\tilde{U}}=\frac{\epsilon \operatorname{Re} B(y)\left(\frac{y_{c}}{y}\right)}{\left[\left(\frac{\Delta y}{\epsilon}\right)^{2}+\left(\frac{\epsilon}{\ell}\right)^{4}\right]^{2}}\left\{-2\left(\frac{\Delta y}{\epsilon}\right)\left(\frac{\epsilon}{\ell}\right)^{2}+\mathrm{i}\left(\left[\left(\frac{\Delta y}{\epsilon}\right)^{2}-\left(\frac{\epsilon}{\ell}\right)^{4}\right]\right)\right\},
$$

which is described in (3.12).

\section{A.3. Inviscid transfer function}

Taking the inviscid (distinguished) limit of (A10) as $R e \rightarrow \infty$ and the critical layer thickness, $\epsilon \rightarrow 0$, assuming that

$$
\lim _{\substack{\epsilon \rightarrow 0 \\ R e \rightarrow \infty}} \epsilon^{3} R e=\left(k_{x} \mathrm{~d} \bar{u}_{c}\right)^{-1}
$$

yields

$$
\frac{\tilde{R}_{x x}}{\tilde{U}}=\left(k_{x} \mathrm{~d} \bar{u}_{c}\right)^{-1}\left\{-\frac{k_{x} A(y)}{\Delta y}+\mathrm{i} \frac{B(y)\left(\frac{y_{c}}{y}\right)}{(\Delta y)^{2}}\right\} .
$$

The distinguished limit follows from the definition of the critical layer thickness itself, (3.1). 


\section{Jacobi, D. Chung, S. Duvvuri and B.J. McKeon}

Note that the sign of the dominant imaginary component is positive, indicating a phase difference between the stress and large scales of $+\pi / 2$, in contrast to the results taking into account the effect of viscosity, where the sign is negative. The phase difference can be written as

$$
\varphi_{i n v}=\arctan \left(\frac{B(y)\left(\frac{y_{c}}{y}\right)}{-k_{x} A(y)(\Delta y)}\right) \text {, }
$$

and its meaning is explained in the context of (3.16) above.

\section{REFERENCES}

Adrian, R.J., Meinhart, C.D. \& Tomkins, C.D. 2000 Vortex organization in the outer region of the turbulent boundary layer. J. Fluid Mech. 422, 1-54.

BaArs, W.J., TAlluru, K.M., Hutchins, N. \& Marusic, I. 2015 Wavelet analysis of wall turbulence to study large-scale modulation of small scales. Exp. Fluids 56 (10), 188.

BAndyopadhyay, P.R. \& Hussain, A.K.M.F. 1984 The coupling between scales in shear flows. Phys. Fluids 27, 2221-2228.

Chernyshenko, S., MARUsic, I. \& MAthis, R. 2012 Quasi-steady description of modulation effects in wall turbulence. arXiv:1203.3714.

Chung, D. \& MCKEON, B.J. 2010 Large-eddy simulation investigation of large-scale structures in a long channel flow. J. Fluid Mech. 661, 341-364.

DAwson, S.T.M. \& MCKEON, B.J. 2019 On the shape of resolvent modes in wall-bounded turbulence. J. Fluid Mech. 877, 682-716.

Duvvuri, S. \& McKeOn, B.J. 2015 Triadic scale interactions in a turbulent boundary layer. J. Fluid Mech. 767, R4.

FERnholZ, H.H. \& Finley, P.J. 1996 The incompressible zero-pressure-gradient turbulent boundary layer: an assessment of the data. Prog. Aerosp. Sci. 32 (4), 245-311.

Ganapathisubramani, B., Hutchins, N., Monty, J.O., Chung, D. \& Marusic, I. 2012 Amplitude and frequency modulation in wall turbulence. J. Fluid Mech. 712, 61-91.

Guala, M., Metzger, M. \& MCKeOn, B.J. 2011 Interactions within the turbulent boundary layer at high Reynolds number. J. Fluid Mech. 666, 573-604.

HABERMAN, R. 1976 Nonlinear perturbations of the Orr/Sommerfeld equation: asymptotic expansion of the logarithmic phase shift across the critical layer. SIAM J. Math. Anal. 7 (1), 70-81.

Hutchins, N. \& Marusic, I. 2007 Large-scale influences in near-wall turbulence. Phil. Trans. R. Soc. Lond. A 365, 647-664.

Hutchins, N., Monty, J.P., Ganapathisubramani, B., NG, H.C.H. \& Marusic, I. 2011 Three-dimensional conditional structure of a high-Reynolds number turbulent boundary layer. J. Fluid Mech. 673, 255-285.

Inoue, M., Mathis, R., Marusic, I. \& Pullin, D.I. 2012 Inner-layer intensities for the flat-plate turbulent boundary layer combining a predictive wall-model with large-eddy simulations. Phys. Fluids 24, 075102.

JACOBI, I. \& MCKeON, B.J. 2013 Phase relationships between large and small scales in the turbulent boundary layer. Exp. Fluids 54, 1481.

JACOBI, I. \& MCKEON, B.J. 2017 Phase-relationships between scales in the perturbed turbulent boundary layer. J. Turbul. 18 (12), 1120-1143.

Kim, K.C. \& AdRiAn, R.J. 1999 Very large-scale motion in the outer layer. Phys. Fluids 11, 417-422.

Lin, C.C. 1955 The Theory of Hydrodynamic Stability. Cambridge University Press.

Luhar, M., Sharma, A.S. \& MCKEON, B.J. 2014 On the structure and origin of pressure fluctuations in wall turbulence: predictions based on the resolvent analysis. J. Fluid Mech. 751, 38-70.

MARUSIC, I. \& HEUER, W.D.C. 2007 Reynolds number invariance of the structural inclination angle in wall turbulence. Phys. Rev. Lett. 99, 114504.

Marusic, I., Mathis, R. \& Hutchins, N. 2010 Predictive model for wall-bounded turbulent flow. Science 329 (5988), 193-196.

Mathis, R., Hutchins, N. \& MARusic, I. 2009a Large-scale amplitude modulation of the small-scale structures in turbulent boundary layers. J. Fluid Mech. 628, 311-337. 


\section{Interactions between scales in wall turbulence}

Mathis, R., Marusic, I., Chernyshenko, S.I. \& Hutchins, N. 2013 Estimating wall-shear-stress fluctuations given an outer region input. J. Fluid Mech. 715, 163-180.

Mathis, R., Marusic, I., Hutchins, N. \& Sreenivasan, K.R. 2011 The relationship between the velocity skewness and the amplitude modulation of the small scale by the large scale in turbulent boundary layers. Phys. Fluids 23, 121702.

Mathis, R., Monty, J.P., Hutchins, N. \& Marusic, I. $2009 b$ Comparison of large-scale amplitude modulation in turbulent boundary layers, pipes and channel flows. Phys. Fluids 21, 111703.

McKeon, B.J. 2017 The engine behind (wall) turbulence: perspectives on scale interactions. J. Fluid Mech. $817, \mathrm{P} 1$.

McKeon, B.J. \& Sharma, A.S. 2010 A critical-layer framework for turbulent pipe flow. J. Fluid Mech. $658,336-382$.

McKeon, B.J., Sharma, A.S. \& JACOBI, I. 2013 Experimental manipulation of wall turbulence: a systems approach. Phys. Fluids 25, 031301.

Morrison, J.F., JiAng, W., MCKeon, B.J. \& Smits, A.J. 2004 Scaling of the streamwise velocity component in turbulent pipe flow. J. Fluid Mech. 508, 99-131.

REYNOLDS, W.C. \& HuSSAIN, A.K.M.F. 1972 The mechanics of an organized wave in turbulent shear flow. Part 3. Theoretical models and comparisons with experiments. J. Fluid Mech. 54, 263-288.

SCHLATTER, P. \& ÖRLÜ, R. 2010 Quantifying the interaction between large and small scales in wall-bounded turbulent flows: a note of caution. Phys. Fluids 22 (5), 051704.

ShARMA, A.S. \& MCKeON, B.J. 2013 On coherent structure in wall turbulence. J. Fluid Mech. 728, 196-238.

Talluru, K.M., Baidya, R., Hutchins, N. \& Marusic, I. 2014 Amplitude modulation of all three velocity components in turbulent boundary layers. J. Fluid Mech. 746, R1.

Vallikivi, M., Ganapathisubramani, B. \& Smits, A.J. 2015 Spectral scaling in boundary layers and pipes at very high Reynolds numbers. J. Fluid Mech. 771, 303-326. 